\title{
Stability and Total Variation Estimates on General Scalar Balance Laws
}

\author{
Rinaldo M. Colombo, Magali Mercier*and Massimiliano D. Rosini ${ }^{\dagger}$ \\ Department of Mathematics, Brescia University \\ Via Branze 38, 25133 Brescia \\ Italy
}

October 14, 2008

\begin{abstract}
Consider the general scalar balance law $\partial_{t} u+\operatorname{Div} f(t, x, u)=F(t, x, u)$ in several space dimensions. The aim of this note is to estimate the dependence of its solutions from the flow $f$ and from the source $F$. To this aim, a bound on the total variation in the space variables of the solution is obtained. This result is then applied to obtain well posedness and stability estimates for a balance law with a non local source.
\end{abstract}

2000 Mathematics Subject Classification: 35L65.

Keywords: Multi-dimensional scalar conservation laws, Kružkov entropy solutions.

\section{Introduction}

The Cauchy problem for a scalar balance law in $N$ space dimension

$$
\left\{\begin{array}{lrl}
\partial_{t} u+\operatorname{Div} f(t, x, u)=F(t, x, u) & (t, x) & \in \mathbb{R}_{+} \times \mathbb{R}^{N} \\
u(0, x)=u_{o}(x) & x & \in \mathbb{R}^{N}
\end{array}\right.
$$

is well known to admit a unique weak entropy solution, as proved in the classical result by Kružkov [12, Theorem 5]. The same paper also provides the basic stability estimate on the dependence of solutions from the initial data, see [12, Theorem 1]. In the same setting established in [12], we provide here an estimate on the dependence of the solutions to (1.1) from the flow $f$, from the source $F$ and recover the known estimate on the dependence from the initial datum $u_{o}$. A key intermediate result is a bound on the total variation of the solution to (1.1), which we provide in Theorem 2.5.

In the case of a conservation law, i.e. $F=0$, and with a flow $f$ independent from $t, x$, the dependence of the solution from $f$ was already considered in [3], where also other results were presented. In this case, the TV bound is obvious, since TV $(u(t)) \leq \mathrm{TV}\left(u_{o}\right)$. The estimate provided by Theorem 2.5 slightly improves the analogous result in [3, Theorem 3.1] (that was already known, see [6, 16]), which reads (for a suitable absolute constant $C$ )

$$
\|u(t)-v(t)\|_{\mathbf{L}^{1}\left(\mathbb{R}^{N} ; \mathbb{R}\right)} \leq\left\|u_{o}-v_{o}\right\|_{\mathbf{L}^{1}\left(\mathbb{R}^{N} ; \mathbb{R}\right)}+C \mathrm{TV}\left(u_{o}\right) \mathbf{L i p}(f-g) t .
$$

\footnotetext{
*Permanent address: Université de Lyon, Université Lyon 1; 43, bd. du 11 novembre 1918; 69622 Villeurbanne Cedex

${ }^{\dagger}$ Supported by INdAM.
} 
Our result, given by Theorem 2.6, reduces to this inequality when $f$ and $g$ are not dependent on $t, x$ and $F=G=0$, but with $C=1$.

An flow dependent also on $x$ was considered in 4 , 9], though in the special case $f(x, u)=$ $l(x) g(u)$, but with a source term containing a possibly degenerate parabolic operator. There, estimates on the $\mathbf{L}^{1}$ distance between solutions in terms of the distance between the flows were obtained, but dependent from an a priori unknown bound on TV $(u(t))$. Here, with no parabolic operators in the source term, we provide fully explicit bounds both on TV $(u(t))$ and on the distance between solutions. Indeed, remark that with no specific assumptions on the flow, TV $(u(t))$ may well blow up to $+\infty$ at $t=0+$, as in the simple case $f(x, u)=\cos x$ with zero initial datum.

Both the total variation and the stability estimates proved below turn out to be optimal in some simple cases, in which optimal estimates are known.

As an example of a possible application, we consider in Section 3 a toy model for a radiating gas. This system was already considered in [5, 8, 10, 11, 13, 14, 15, 17]. It consists of a balance law of the type (1.1), but with a source that contains also a non local term, due to the convolution of the unknown with a suitable kernel. Thanks to the present results, we prove the well posedness of the model extending [8, Theorem 2.4] to more general flows, sources and convolution kernels. Stability and total variation estimates are also provided.

This paper is organized as follows: in Section 2, we introduce the notation, state the main results and compare them with those found in the literature. Section 3 is devoted to an application to a radiating gas model. Finally, in sections 1 and 5 the detailed proofs of theorems 2.5 and 2.6 are provided.

\section{Notation and Main Results}

Denote $\overline{\mathbb{R}}_{+}=\left[0,+\infty\left[\right.\right.$ and $\left.\mathbb{R}_{+}=\right] 0,+\infty\left[\right.$. Below, $N$ is a positive integer, $\Omega=\mathbb{R}_{+} \times \mathbb{R}^{N} \times \mathbb{R}$, $B(x, r)$ denotes the ball in $\mathbb{R}^{N}$ with center $x \in \mathbb{R}^{N}$ and radius $r>0$. The volume of the unit ball $B(0,1)$ is $\omega_{N}$. For notational simplicity, we set $\omega_{0}=1$. The following relation can be proved using the expression of $\omega_{N}$ in terms of the Wallis integral $W_{N}$ :

$$
\frac{\omega_{N}}{\omega_{N-1}}=2 W_{N} \quad \text { where } \quad W_{N}=\int_{0}^{\pi / 2}(\cos \theta)^{N} \mathrm{~d} \theta .
$$

In the present work, $\mathbf{1}_{A}$ is the characteristic function of the set $A$ and $\delta_{t}$ is the Dirac measure centered at $t$. Besides, for a vector valued function $f=f(x, u)$ with $u=u(x)$, $\operatorname{Div} f$ stands for the total divergence. On the other hand, $\operatorname{div} f$, respectively $\nabla f$, denotes the partial divergence, respectively gradient, with respect to the space variables. Moreover, $\partial_{u}$ and $\partial_{t}$ are the usual partial derivatives. Thus, $\operatorname{Div} f=\operatorname{div} f+\partial_{u} f \cdot \nabla u$.

Recall the definition of weak entropy solution to (1.1), see [12, Definition 1].

Definition 2.1 A function $u \in \mathbf{L}^{\infty}\left(\mathbb{R}_{+} \times \mathbb{R}^{N} ; \mathbb{R}\right)$ is a weak entropy solution to (1.1) if: 1. for any constant $k \in \mathbb{R}$ and any test function $\varphi \in \mathbf{C}_{\mathbf{c}}^{\infty}\left(\mathbb{R}_{+} \times \mathbb{R}^{N} ; \overline{\mathbb{R}}_{+}\right)$

$$
\begin{gathered}
\int_{\mathbb{R}_{+}} \int_{\mathbb{R}^{N}}\left[(u-k) \partial_{t} \varphi+(f(t, x, u)-f(t, x, k)) \cdot \nabla \varphi+(F(t, x, u)-\operatorname{div} f(t, x, k)) \varphi\right] \\
\times \operatorname{sign}(u-k) \mathrm{d} x \mathrm{~d} t \geq 0
\end{gathered}
$$


2. there exists a set $\mathcal{E}$ of zero measure in $\overline{\mathbb{R}}_{+}$such that for $t \in \overline{\mathbb{R}}_{+} \backslash \mathcal{E}$ the function $u(t, x)$ is defined almost everywhere in $\mathbb{R}^{N}$ and for any $r>0$

$$
\lim _{t \rightarrow 0, t \in \overline{\mathbb{R}}_{+} \backslash \mathcal{E}} \int_{B(0, r)}\left|u(t, x)-u_{o}(x)\right| \mathrm{d} x=0 .
$$

Throughout this paper, we refer to [1, 18] as general references for the theory of BV functions. In particular, recall the following basic definition, see [1, Definition 3.4 and Theorem 3.6].

Definition 2.2 Let $u \in \mathbf{L}_{\text {loc }}^{1}\left(\mathbb{R}^{N} ; \mathbb{R}\right)$. Define

$$
\begin{aligned}
\operatorname{TV}(u) & =\sup \left\{\int_{\mathbb{R}^{N}} u \operatorname{div} \psi \mathrm{d} x: \psi \in \mathbf{C}_{\mathbf{c}}^{\mathbf{1}}\left(\mathbb{R}^{N} ; \mathbb{R}^{N}\right) \text { and }\|\psi\|_{\mathbf{L}^{\infty}\left(\mathbb{R}^{N} ; \mathbb{R}^{N}\right)} \leq 1\right\} \\
\mathbf{B V}\left(\mathbb{R}^{N} ; \mathbb{R}\right) & =\left\{u \in \mathbf{L}_{\mathbf{l o c}}^{\mathbf{1}}\left(\mathbb{R}^{N} ; \mathbb{R}\right): \operatorname{TV}(u)<+\infty\right\} .
\end{aligned}
$$

The following sets of assumptions will be of use below.

(H1)

$$
\begin{cases}f \in \mathbf{C}^{2}\left(\Omega ; \mathbb{R}^{N}\right) & F \in \mathbf{C}^{\mathbf{1}}(\Omega ; \mathbb{R}) \\ \partial_{u} f \in \mathbf{L}^{\infty}\left(\Omega ; \mathbb{R}^{N}\right) & \\ \partial_{u}(F-\operatorname{div} f) \in \mathbf{L}^{\infty}(\Omega ; \mathbb{R}) & F-\operatorname{div} f \in \mathbf{L}^{\infty}(\Omega ; \mathbb{R})\end{cases}
$$

(H2) $\begin{cases}f \in \mathbf{C}^{2}\left(\Omega ; \mathbb{R}^{N}\right) & F \in \mathbf{C}^{\mathbf{1}}(\Omega ; \mathbb{R}) \\ \nabla \partial_{u} f \in \mathbf{L}^{\infty}\left(\Omega ; \mathbb{R}^{N \times N}\right) & \int_{\mathbb{R}_{+}} \int_{\mathbb{R}^{N}}\|\nabla(F-\operatorname{div} f)(t, x, \cdot)\|_{\mathbf{L}^{\infty}\left(\mathbb{R} ; \mathbb{R}^{N}\right)} \mathrm{d} x \mathrm{~d} t<+\infty \\ \partial_{t} \partial_{u} f \in \mathbf{L}^{\infty}\left(\Omega ; \mathbb{R}^{N}\right) & \partial_{t} F \in \mathbf{L}^{\infty}(\Omega ; \mathbb{R}) \\ \partial_{t} \operatorname{div} f \in \mathbf{L}^{\infty}(\Omega ; \mathbb{R}) & \end{cases}$

(H3) $\begin{cases}f \in \mathbf{C}^{\mathbf{1}}\left(\Omega ; \mathbb{R}^{N}\right) & F \in \mathbf{C}^{\mathbf{0}}(\Omega ; \mathbb{R}) \quad \partial_{u} F \in \mathbf{L}^{\infty}(\Omega ; \mathbb{R}) \\ \partial_{u} f \in \mathbf{L}^{\infty}\left(\Omega ; \mathbb{R}^{N}\right) & \int_{\mathbb{R}_{+}} \int_{\mathbb{R}^{N}}\|(F-\operatorname{div} f)(t, x, \cdot)\|_{\mathbf{L}^{\infty}(\mathbb{R} ; \mathbb{R})} \mathrm{d} x \mathrm{~d} t<+\infty\end{cases}$

The quantity $F-\operatorname{div} f$ has a particular role, since it behaves as the "true" source, see (2.6). We note there that the assumptions above can be significantly softened in various specific situations. For instance, the requirement that $f$ be Lipschitz, which is however a standard hypothesis, see [3, Paragraph 3], can be relaxed to $f$ locally Lipschitz in the case $f=$ $f(u)$ and $F=0$, thanks to the maximum principle [12, Theorem 3]. Furthermore, the assumptions above can be obviously weakened when aiming at estimates on bounded time intervals.

Assumptions (H1) are those used in the classical results [12, Theorem 1 and Theorem 5]. However, we stress that the proofs below need less regularity. As in [12], we remark that no derivative of $f$ or $F$ in time is ever needed. Furthermore, $f$ needs not be twice differentiable in $u$, for the only second derivatives required are $\nabla_{x} \partial_{u} f$ and $\nabla_{x}^{2} f$.

We recall below the classical result by Kružkov.

Theorem 2.3 (Kružkov) Let (H1) hold. Then, for any $u_{o} \in \mathbf{L}^{\infty}\left(\mathbb{R}^{N} ; \mathbb{R}\right)$, there exists a unique weak entropy solution $u$ to 1.1$)$ in $\mathbf{L}^{\infty}\left(\overline{\mathbb{R}}_{+} ; \mathbf{L}_{\text {loc }}^{1}\left(\mathbb{R}^{N} ; \mathbb{R}\right)\right)$ continuous from the right. Moreover, if a sequence $u_{o}^{n} \in \mathbf{L}^{\infty}\left(\mathbb{R}^{N} ; \mathbb{R}\right)$ converges to $u_{o}$ in $\mathbf{L}_{\text {loc }}^{1}$, then for all $t>0$ the corresponding solutions $u^{n}(t)$ converge to $u(t)$ in $\mathbf{L}_{\text {loc }}^{1}$. 
Remark 2.4 Under the conditions (H2) and $\int_{\mathbb{R}_{+}} \int_{\mathbb{R}^{N}}\|(F-\operatorname{div} f)(t, x, \cdot)\|_{\mathbf{L}^{\infty}(\mathbb{R} ; \mathbb{R})} \mathrm{d} x \mathrm{~d} t<$ $+\infty$, see (H3), the estimate provided by Theorem 2.5 below, allows to use the technique described in [7, Theorem 4.3.1], proving the continuity in time of the solution, so that $u \in \mathbf{C}^{\mathbf{0}}\left(\overline{\mathbb{R}}_{+} ; \mathbf{L}_{\text {loc }}^{\mathbf{1}}\left(\mathbb{R}^{N} ; \mathbb{R}\right)\right)$.

\subsection{Estimate on the Total Variation}

Recall that [9, Theorem 1.3] and [4, Theorem 3.2] provide stability bounds on (1.1), in the more general case with a degenerate parabolic source, but assuming a priori bounds on the total variation of solutions. Our first result provides these bounds.

Theorem 2.5 Assume that (H1) and (H2) hold. Let $u_{o} \in \mathbf{B V}\left(\mathbb{R}^{N} ; \mathbb{R}\right)$. Then, the weak entropy solution $u$ of (1.1) satisfies $u(t) \in \mathbf{B V}\left(\mathbb{R}^{N} ; \mathbb{R}\right)$ for all $t>0$. Moreover, let

$$
\kappa_{o}=N W_{N}\left((2 N+1)\left\|\nabla \partial_{u} f\right\|_{\mathbf{L}^{\infty}\left(\Omega ; \mathbb{R}^{N \times N}\right)}+\left\|\partial_{u} F\right\|_{\mathbf{L}^{\infty}(\Omega ; \mathbb{R})}\right)
$$

with $W_{N}$ as in (2.1). Then, for all $T>0$,

$$
\operatorname{TV}(u(T)) \leq \operatorname{TV}\left(u_{o}\right) e^{\kappa_{o} T}+N W_{N} \int_{0}^{T} e^{\kappa_{o}(T-t)} \int_{\mathbb{R}^{N}}\|\nabla(F-\operatorname{div} f)(t, x, \cdot)\|_{\mathbf{L}^{\infty}} \mathrm{d} x \mathrm{~d} t .
$$

This estimate is optimal in the following situations:

1. If $f$ is independent from $x$ and $F=0$, then $\kappa_{o}=0$ and the integrand in the right hand side above vanishes. Hence, (2.5) reduces to the well known optimal bound $\mathrm{TV}(u(t)) \leq \mathrm{TV}\left(u_{o}\right)$.

2. In the $1 \mathrm{D}$ case, if $f$ and $F$ are both independent from $t$ and $u$, then $\kappa_{o}=0$ and (1.1) reduces to the ordinary differential equation $\partial_{t} u=F-\operatorname{div} f$. In this case, 2.5 becomes

$$
\mathrm{TV}(u(t)) \leq \operatorname{TV}\left(u_{o}\right)+t \operatorname{TV}(F-\operatorname{div} f) .
$$

3. If $f=0$ and $F=F(t)$ then, trivially, $\mathrm{TV}(u(t))=\mathrm{TV}\left(u_{o}\right)$ and (2.5) is optimal.

A simpler but slightly weaker form of (2.5) is

$$
\operatorname{TV}(u(T)) \leq \operatorname{TV}\left(u_{o}\right) e^{\kappa_{o} T}+N W_{N} \frac{e^{\kappa_{o} T}-1}{\kappa_{o}} \sup _{t \in[0, T]} \int_{\mathbb{R}^{N}}\|\nabla(F-\operatorname{div} f)(t, x, \cdot)\|_{\mathbf{L}^{\infty}} \mathrm{d} x
$$

when the right hand side is bounded.

\subsection{Stability of Solutions with Respect to Flow and Source}

Consider now (1.1) together with the analogous problem

$$
\left\{\begin{array}{lrl}
\partial_{t} v+\operatorname{Div} g(t, x, v)=G(t, x, v) & (t, x) & \in \mathbb{R}_{+} \times \mathbb{R}^{N} \\
v(0, x)=v_{o}(x) & x & \in \mathbb{R}^{N} .
\end{array}\right.
$$

We aim at estimates for the difference $u-v$ between the solutions in terms of $f-g$, $F-G$ and $u_{o}-v_{o}$. Estimates of this type were derived by Bouchut \& Perthame in [3] when $f, g$ depend only on $u$ and $F=G=0$. Here, we generalize their result adding the $(t, x)$-dependence. The present technique is essentially based on Theorem 2.5. 
Theorem 2.6 Let $(f, F),(g, G)$ verify (H1), $(f, F)$ verify (H2) and $(f-g, F-G)$ verify (H3). Let $u_{o}, v_{o} \in \mathbf{B V}\left(\mathbb{R}^{N} ; \mathbb{R}\right)$. We denote $\kappa_{o}$ as in (2.4) and introduce $\kappa=2 N\left\|\nabla \partial_{u} f\right\|_{\mathbf{L}^{\infty}\left(\Omega ; \mathbb{R}^{N \times N}\right)}+\left\|\partial_{u} F\right\|_{\mathbf{L}^{\infty}(\Omega ; \mathbb{R})}+\left\|\partial_{u}(F-G)\right\|_{\mathbf{L}^{\infty}(\Omega ; \mathbb{R})}$ and $M=\left\|\partial_{u} g\right\|_{\mathbf{L}^{\infty}\left(\Omega ; \mathbb{R}^{N}\right)}$. Then, for any $T, R>0$ and $x_{o} \in \mathbb{R}^{N}$, the following estimate holds:

$$
\begin{aligned}
& \int_{\left\|x-x_{o}\right\| \leq R}|u(T, x)-v(T, x)| \mathrm{d} x \leq e^{\kappa T} \int_{\left\|x-x_{o}\right\| \leq R+M T}\left|u_{o}(x)-v_{o}(x)\right| \mathrm{d} x \\
+ & \frac{e^{\kappa_{o} T}-e^{\kappa T}}{\kappa_{o}-\kappa} \operatorname{TV}\left(u_{o}\right)\left\|\partial_{u}(f-g)\right\|_{\mathbf{L}^{\infty}} \\
+ & N W_{N}\left(\int_{0}^{T} \frac{e^{\kappa_{o}(T-t)}-e^{\kappa(T-t)}}{\kappa_{o}-\kappa} \int_{\mathbb{R}^{N}}\|\nabla(F-\operatorname{div} f)(t, x, \cdot)\|_{\mathbf{L}^{\infty}} \mathrm{d} x \mathrm{~d} t\right)\left\|\partial_{u}(f-g)\right\|_{\mathbf{L}^{\infty}} \\
+ & \int_{0}^{T} e^{\kappa(T-t)} \int_{\left\|x-x_{o}\right\| \leq R+M(T-t)}\|((F-G)-\operatorname{div}(f-g))(t, x, \cdot)\|_{\mathbf{L}^{\infty}} \mathrm{d} x \mathrm{~d} t .
\end{aligned}
$$

The above inequality is undefined for $\kappa=\kappa_{o}$ and, in this case, it reduces to (5.17). This bound is optimal in the following situations, where $u_{o}, v_{o} \in \mathbf{L}^{\mathbf{1}}\left(\mathbb{R}^{N} ; \mathbb{R}\right)$.

1. In the standard case of a conservation law, i.e. when $F=G=0$ and $f, g$ are independent of $x$, we have $\kappa_{o}=\kappa=0$ and the result of Theorem 2.6 becomes, see 2 , Theorem 2.1],

$$
\|u(T)-v(T)\|_{\mathbf{L}^{1}\left(\mathbb{R}^{N} ; \mathbb{R}\right)} \leq\left\|u_{o}-v_{o}\right\|_{\mathbf{L}^{1}\left(\mathbb{R}^{n} ; \mathbb{R}\right)}+T \operatorname{TV}\left(u_{o}\right)\left\|\partial_{u}(f-g)\right\|_{\mathbf{L}^{\infty}\left(\Omega ; \mathbb{R}^{N}\right)} .
$$

2. If $\partial_{u} f=\partial_{u} g=0$ and $\partial_{u} F=\partial_{u} G=0$, then $\kappa_{o}=\kappa=0$ and Theorem 2.6 now reads

$$
\|u(T)-v(T)\|_{\mathbf{L}^{\mathbf{1}\left(\mathbb{R}^{N} ; \mathbb{R}\right)}} \leq\left\|u_{o}-v_{o}\right\|_{\mathbf{L}^{\mathbf{1}\left(\mathbb{R}^{N} ; \mathbb{R}\right)}}+\int_{0}^{T}\|[(F-G)-\operatorname{div}(f-g)](t)\|_{\mathbf{L}^{\mathbf{1}\left(\mathbb{R}^{N} ; \mathbb{R}\right)}} \mathrm{d} t .
$$

3. If $(f, F)$ and $(g, G)$ are dependent only on $x$, then Theorem 2.6 reduces to

$$
\|u(T)-v(T)\|_{\mathbf{L}^{1}\left(\mathbb{R}^{N} ; \mathbb{R}\right)} \leq\left\|u_{o}-v_{o}\right\|_{\mathbf{L}^{1}\left(\mathbb{R}^{N} ; \mathbb{R}\right)}+T\|(F-G)-\operatorname{div}(f-g)\|_{\mathbf{L}^{1}\left(\mathbb{R}^{N} ; \mathbb{R}\right)} .
$$

The estimate obtained in Theorem 2.6 shows also that, depending on the properties of specific applications, the regularity requirement $f \in \mathbf{C}^{2}\left(\Omega ; \mathbb{R}^{N}\right)$ can be significantly relaxed. For instance, in the case $f(t, x, u)=q(u) v(x)$ considered in [4, 9, asking $q$ of class $\mathbf{C}^{\mathbf{1}}$ and $v$ of class $\mathbf{C}^{2}$ is sufficient. See also Section 3 for a case in which the required regularity in time can be reduced.

In the case of conservations laws, i.e. when $F=G=0$, one proves that $\kappa<\kappa_{o}$ and the estimate in Theorem 2.6 takes the somewhat simpler form

$$
\begin{aligned}
& \int_{\left\|x-x_{o}\right\| \leq R}|u(T, x)-v(T, x)| \mathrm{d} x \leq e^{\kappa T} \int_{\left\|x-x_{o}\right\| \leq R+M T}\left|u_{o}(x)-v_{o}(x)\right| \mathrm{d} x \\
+ & T e^{\kappa_{o} T} \operatorname{TV}\left(u_{o}\right)\left\|\partial_{u}(f-g)\right\|_{\mathbf{L}^{\infty}} \\
+ & N W_{N} T^{2} e^{\kappa_{o} T} \sup _{t \in[0, T]}\left(\int_{\mathbb{R}^{N}}\|\nabla \operatorname{div} f(t, x, \cdot)\|_{\mathbf{L}^{\infty}} \mathrm{d} x\right)\left\|\partial_{u}(f-g)\right\|_{\mathbf{L}^{\infty}} \\
+ & T e^{\kappa_{o} T} \sup _{t \in[0, T]} \int_{\left\|x-x_{o}\right\| \leq R+M(T-t)}\|\operatorname{div}(f-g)(t, x, \cdot)\|_{\mathbf{L}^{\infty}} \mathrm{d} x
\end{aligned}
$$

when the right hand side is bounded. In the case considered in [3, Theorem 3.1], $f=f(u)$, $\kappa_{o}=0$ and we obtain [3, formula (3.2)] with 1 instead of the constant $C$ therein. 


\section{Application to a Radiating Gas Model}

The following balance law is a toy model inspired by Euler equations for radiating gases:

$$
\partial_{t} u+\operatorname{Div} f(t, x, u)=-u+K *_{x} u .
$$

It has been extensively studied in the literature when $f=f(u)$, see for instance 10, 11, 13, 15, 17] for the scalar 1D case, [5, 14] for 1D systems, [8] for the scalar ND case.

The estimate provided by Theorem 2.6 allows us to present an alternative proof of the well posedness of (3.1) proved in [8]. Furthermore, we add stability estimates on the dependence of the solution from $f$ and $K$, in the case of $f$ dependent also on $t, x$ and with more general source terms.

Theorem 3.1 Let $(f, F)$ satisfy (H1), (H2) and (H3). Assume that

$$
K \in\left(\mathbf{C}^{\mathbf{2}} \cap \mathbf{L}^{\infty}\right)\left(\mathbb{R}_{+} \times \mathbb{R}^{N} ; \mathbb{R}\right) \quad \text { and } \quad K \in \mathbf{L}^{\infty}\left(\mathbb{R}_{+} ; \mathbf{W}^{\mathbf{2}, \mathbf{1}}\left(\mathbb{R}^{N} ; \mathbb{R}\right)\right) .
$$

Then, for any $u_{o} \in\left(\mathbf{B V} \cap \mathbf{L}^{\mathbf{1}}\right)\left(\mathbb{R}^{N} ; \mathbb{R}\right)$, the Cauchy problem

$$
\left\{\begin{array}{lrl}
\partial_{t} u+\operatorname{Div} f(t, x, u)=F(t, x, u)+K *_{x} u & (t, x) & \in \mathbb{R}_{+} \times \mathbb{R}^{N} \\
u(0, x)=u_{o}(x) & x & \in \mathbb{R}^{N}
\end{array}\right.
$$

admits a unique weak entropy solution $u \in \mathbf{C}^{\mathbf{0}}\left(\overline{\mathbb{R}}_{+} ; \mathbf{L}^{\mathbf{1}}\left(\mathbb{R}^{N} ; \mathbb{R}\right)\right)$. Moreover, denoting $k=\|K\|_{\mathbf{L}^{\infty}\left(\mathbb{R}_{+} ; \mathbf{L}^{\mathbf{1}}\left(\mathbb{R}^{N} ; \mathbb{R}\right)\right)}$, for all $T>0$, the following estimate holds:

$$
\begin{aligned}
\operatorname{TV}(u(T)) \leq & e^{\left(\kappa_{o}+N W_{N} k\right) T} \operatorname{TV}\left(u_{o}\right) \\
& +N W_{N} \int_{0}^{T} e^{\left(\kappa_{o}+N W_{N} k\right)(T-t)} \int_{\mathbb{R}^{N}}\|\nabla(F-\operatorname{div} f)(t, x, \cdot)\|_{\mathbf{L}^{\infty}} \mathrm{d} x \mathrm{~d} t .
\end{aligned}
$$

If $F(t, x, 0)-\operatorname{div} f(t, x, 0)=0$ for all $t \in[0, T]$ and $x \in \mathbb{R}^{N}$, then

1. $\|u(T)\|_{\mathbf{L}^{1}\left(\mathbb{R}^{N} ; \mathbb{R}\right)} \leq e^{(\kappa+k) T}\left\|u_{o}\right\|_{\mathbf{L}^{1}\left(\mathbb{R}^{N} ; \mathbb{R}\right)}$.

2. Let $\tilde{K}$ satisfy $(\boldsymbol{K})$ and call $\tilde{u}$ the solution to (3.9) with $K$ replaced by $\tilde{K}$. Then,

$$
\|u(T)-\tilde{u}(T)\|_{\mathbf{L}^{1}\left(\mathbb{R}^{N} ; \mathbb{R}\right)} \leq\left\|u_{o}\right\|_{\mathbf{L}^{1}\left(\mathbb{R}^{N} ; \mathbb{R}\right)} \frac{e^{k T}-e^{\tilde{k} T}}{k-\tilde{k}}\|K-\tilde{K}\|_{\mathbf{L}^{\infty}\left(\mathbb{R}_{+} ; \mathbf{L}^{1}\left(\mathbb{R}^{N} ; \mathbb{R}\right)\right)}
$$

Proof. Fix a positive $T$ (to be specified below) and consider the Banach space $X=$ $\mathbf{C}^{\mathbf{0}}\left([0, T] ; \mathbf{L}^{\mathbf{1}}\left(\mathbb{R}^{N} ; \mathbb{R}\right)\right)$ equipped with the usual norm $\|u\|_{X}=\|u\|_{\mathbf{L}^{\infty}\left(\mathbb{R}_{+} ; \mathbf{L}^{\mathbf{1}}\left(\mathbb{R}^{N} ; \mathbb{R}\right)\right)}$. Define on $X$ the map $\mathcal{T}$ so that $\mathcal{T}(w)=u$ if and only if $u$ solves

$$
\left\{\begin{array}{lrl}
\partial_{t} u+\operatorname{Div} f(t, x, u)=F(t, x, u)+K *_{x} w & (t, x) & \in \mathbb{R}_{+} \times \mathbb{R}^{N} \\
u(0, x)=u_{o}(x) & x & \in \mathbb{R}^{N}
\end{array}\right.
$$

in the sense of Definition 2.1. Note that the source term does not have the regularity required in (H1). However, by the estimate in Theorem 2.6, we can prove that (3.4) does indeed have a unique weak entropy solution, see Lemma 3.2 for the details. The fixed 
points of $\mathcal{T}$ are the solutions to (3.1). By Theorem 2.3 and Remark 2.4, $\mathcal{T} w \in X$ for all $w \in X$. We now show that $\mathcal{T}$ is a contraction, provided $T$ is sufficiently small. Note that

$$
\begin{aligned}
\kappa_{o} & =N W_{N}\left((2 N+1)\left\|\nabla \partial_{u} f\right\|_{\mathbf{L}^{\infty}}+\left\|\partial_{u} F\right\|_{\mathbf{L}^{\infty}}\right) \\
\kappa & =2 N\left\|\nabla \partial_{u} f\right\|_{\mathbf{L}^{\infty}}+\left\|\partial_{u} F\right\|_{\mathbf{L}^{\infty}} .
\end{aligned}
$$

Moreover, by Theorem 2.6

$$
\begin{aligned}
d\left(\mathcal{T} w_{1}, \mathcal{T} w_{2}\right) & =\sup _{t \in[0, T]}\left\|\mathcal{T} w_{1}-\mathcal{T} w_{2}\right\|_{\mathbf{L}^{1}} \\
& \leq \sup _{t \in[0, T]}\left(\frac{e^{\kappa t}-1}{\kappa} \sup _{\tau \in[0, t]}\left\|K(\tau) *_{x}\left(w_{1}-w_{2}\right)(\tau)\right\|_{\mathbf{L}^{1}}\right) \\
& \leq \frac{e^{\kappa T}-1}{\kappa} \sup _{\tau \in[0, T]}\|K(\tau)\|_{\mathbf{L}^{1}}\left\|\left(w_{1}-w_{2}\right)(\tau)\right\|_{\mathbf{L}^{1}} \\
& \leq \frac{e^{\kappa T}-1}{\kappa} k d\left(w_{1}, w_{2}\right) .
\end{aligned}
$$

Therefore, $\mathcal{T}$ is a contraction as soon as $T$ is smaller than a threshold that depends only on $\left\|\partial_{u} F\right\|_{\mathbf{L}^{\infty}(\Omega: \mathbb{R})},\left\|\nabla \partial_{u} f\right\|_{\mathbf{L}^{\infty}\left(\Omega: \mathbb{R}^{N \times N}\right)}$ and on $\|K\|_{\mathbf{L}^{\infty}\left(\mathbb{R}_{+} ; \mathbf{L}^{1}\left(\mathbb{R}^{N} ; \mathbb{R}\right)\right)}$. Therefore, we proved the well posedness of (3.2) globally in time.

Consider the bound on TV $(u(t))$. By Theorem 2.5,

$$
\begin{aligned}
\operatorname{TV}(u(T)) \leq & \operatorname{TV}\left(u_{o}\right)+N W_{N} \int_{0}^{T} e^{\kappa_{o}(T-t)} \int_{\mathbb{R}^{N}}\|\nabla(F-\operatorname{div} f)(t, x, \cdot)\|_{\mathbf{L}^{\infty}\left(\mathbb{R} ; \mathbb{R}^{N}\right)} \mathrm{d} x \mathrm{~d} t \\
& +N W_{N} \int_{0}^{T} e^{\kappa_{o}(T-t)} k \operatorname{TV}(u(t)) \mathrm{d} t
\end{aligned}
$$

and an application of Gronwall Lemma gives the desired bound.

We estimate the $\mathbf{L}^{\mathbf{1}}$ norm of the solution to (3.2), comparing it with the solution to

$$
\left\{\begin{array}{lrl}
\partial_{t} u+\operatorname{Div} f(t, x, u)=F(t, x, u)+K *_{x} u & (t, x) & \in \mathbb{R}_{+} \times \mathbb{R}^{N} \\
u(0, x)=0 & x & \in \mathbb{R}^{N} .
\end{array}\right.
$$

By assumption, 0 solves (3.5), hence it is its unique solution. Then, evaluating the distance between the solutions of (3.2) and (3.5) by means of Theorem 2.6, we get

$$
e^{-\kappa T}\|u(T)\|_{\mathbf{L}^{1}\left(\mathbb{R}^{N} ; \mathbb{R}\right)} \leq\left\|u_{o}\right\|_{\mathbf{L}^{1}\left(\mathbb{R}^{N} ; \mathbb{R}\right)}+\int_{0}^{T} e^{-\kappa t} \int_{\mathbb{R}^{N}}\left|K *_{x} u(t, x)\right| \mathrm{d} x \mathrm{~d} t
$$

and, thanks to Gronwall Lemma, we obtain:

$$
\|u(T)\|_{\mathbf{L}^{\mathbf{1}\left(\mathbb{R}^{N} ; \mathbb{R}\right)}} \leq e^{(\kappa+k) T}\left\|u_{o}\right\|_{\mathbf{L}^{\mathbf{1}}\left(\mathbb{R}^{N} ; \mathbb{R}\right)} .
$$

The final estimate (3.3) follows from Theorem 2.6:

$$
\begin{aligned}
& e^{-\kappa T}\|(u-\tilde{u})(T)\|_{\mathbf{L}^{1}\left(\mathbb{R}^{N} ; \mathbb{R}\right)} \\
\leq & \|K-\tilde{K}\|_{\mathbf{L}^{\infty}\left(\mathbb{R}_{+} ; \mathbf{L}^{\left.\mathbf{1}\left(\mathbb{R}^{N} ; \mathbb{R}\right)\right)}\right.} \int_{0}^{T} e^{-\kappa t}\|u(t)\|_{\mathbf{L}^{1}\left(\mathbb{R}^{N} ; \mathbb{R}\right)} \mathrm{d} t+k \int_{0}^{T} e^{-\kappa t}\|(u-\tilde{u})(t)\|_{\mathbf{L}^{\mathbf{1}\left(\mathbb{R}^{N} ; \mathbb{R}\right)}} \mathrm{d} t \\
\leq & \|K-\tilde{K}\|_{\mathbf{L}^{\infty\left(\mathbb{R}_{+} ; \mathbf{L}^{1}\left(\mathbb{R}^{N} ; \mathbb{R}\right)\right)}}\left\|u_{o}\right\|_{\mathbf{L}^{\mathbf{1}\left(\mathbb{R}^{N} ; \mathbb{R}\right)}} \frac{e^{k T}-1}{k}+\tilde{k} \int_{0}^{T} e^{-\kappa t}\|(u-\tilde{u})(t)\|_{\mathbf{L}^{\mathbf{1}\left(\mathbb{R}^{N} ; \mathbb{R}\right)}} \mathrm{d} t
\end{aligned}
$$

and thanks to Gronwall Lemma, we get the result.

The continuity in time is proved as described in Remark 2.4. 
Lemma 3.2 Let $f, F$ satisfy (H1) and $K$ satisfy $(\boldsymbol{K})$. If $w \in \mathbf{L}^{\infty}\left(\overline{\mathbb{R}}_{+} \times \mathbb{R}^{N} ; \mathbb{R}\right)$, then the estimates in Theorem 2.5 and in Theorem 2.0 apply also to (3.4).

Proof. Fix positive $T, R$ and let $w_{n}$ be a sequence of $\mathbf{C}^{\infty}$ functions converging to $w$ in $\mathbf{L}^{\mathbf{1}}\left([0, T] \times \mathbb{R}^{N} ; \mathbb{R}\right)$. Apply Theorem 2.3 to the approximate problem

$$
\left\{\begin{array}{lrl}
\partial_{t} u+\operatorname{Div} f(t, x, u)=F(t, x, u)+K *_{x} w_{n} & (t, x) & \in \mathbb{R}_{+} \times \mathbb{R}^{N} \\
u(0, x)=u_{o}(x) & x & \in \mathbb{R}^{N}
\end{array}\right.
$$

to ensure the existence of its weak entropy solution $u_{n}$. Apply Theorem 2.6 to estimate the distance between $u_{n}$ and $u_{n-1}$ :

$$
\begin{aligned}
\left\|u_{n}-u_{n-1}\right\|_{\mathbf{L}^{\infty}\left([0, T] ; \mathbf{L}^{\mathbf{1}}\left(\mathbb{R}^{N} ; \mathbb{R}\right)\right)} & \leq \int_{0}^{T} e^{\kappa(T-t)} \int_{\mathbb{R}^{N}}\left|K *\left(w_{n}-w_{n-1}\right)(t, x)\right| \mathrm{d} x \mathrm{~d} t \\
& \leq e^{\kappa T} k\left\|w_{n}-w_{n-1}\right\|_{\mathbf{L}^{\mathbf{1}}\left([0, T] \times \mathbb{R}^{N} ; \mathbb{R}\right)}
\end{aligned}
$$

showing that the $u_{n}$ form a Cauchy sequence. Their limit $u$ solves (3.2), as it follows passing to the limit over $n$ in the integral conditions (2.2) $-(2.3)$ and applying the Dominated Convergence Theorem. The estimates in theorems 2.5 and 2.6 are extended similarly.

\section{Proof of Theorem 2.5}

Lemma 4.1 Fix a function $\mu_{1} \in \mathbf{C}_{\mathbf{c}}^{\infty}\left(\overline{\mathbb{R}}_{+} ; \overline{\mathbb{R}}_{+}\right)$with

$$
\operatorname{supp}\left(\mu_{1}\right) \subseteq\left[0,1\left[, \quad \int_{\mathbb{R}_{+}} r^{N-1} \mu_{1}(r) \mathrm{d} r=\frac{1}{N \omega_{N}}, \quad \mu_{1}^{\prime} \leq 0, \quad \mu_{1}^{(n)}(0)=0 \text { for } n \geq 1 .\right.\right.
$$

Define

$$
\mu(x)=\frac{1}{\lambda^{N}} \mu_{1}\left(\frac{\|x\|}{\lambda}\right) .
$$

Then, recalling that $\omega_{0}=1$,

$$
\begin{aligned}
\int_{\mathbb{R}^{N}} \mu(x) \mathrm{d} x & =1 \\
\int_{\mathbb{R}^{N}}\left|x_{1}\right| \mu_{1}(\|x\|) \mathrm{d} x & =\frac{2}{N} \frac{\omega_{N-1}}{\omega_{N}} \int_{\mathbb{R}^{N}}\|x\| \mu_{1}(\|x\|) \mathrm{d} x, \\
\int_{\mathbb{R}^{N}}\|x\|\|\nabla \mu(x)\| \mathrm{d} x & =-\int_{\mathbb{R}^{N}}\|x\| \mu_{1}^{\prime}(\|x\|) \mathrm{d} x=N, \\
\int_{\mathbb{R}^{N}}\|x\|^{2} \mu_{1}^{\prime}(\|x\|) \mathrm{d} x & =-(N+1) \int_{\mathbb{R}^{N}}\|x\| \mu_{1}(\|x\|) \mathrm{d} x .
\end{aligned}
$$

Proof. The first relation is immediate. Equalities (4.5) and (4.6) follow directly from an integration by parts. Consider (4.4). The cases $N=1,2,3$ follow from direct computations. Let $N \geq 4$ and pass to spherical coordinates $\left(\rho, \theta_{1}, \ldots, \theta_{N-1}\right)$,

$$
x_{1}=\rho \cos \theta_{N-1}
$$




$$
\begin{aligned}
x_{2} & =\rho \sin \theta_{N-1} \cos \theta_{N-2} \\
\vdots & \vdots \\
x_{N-1} & =\rho \sin \theta_{N-1} \sin \theta_{N-2} \cdots \cos \theta_{1} \\
x_{N} & =\rho \sin \theta_{N-1} \sin \theta_{N-2} \cdots \sin \theta_{1}
\end{aligned}
$$

with $\rho \in \mathbb{R}_{+}, \theta_{1} \in\left[0,2 \pi\left[\right.\right.$ and $\theta_{j} \in[0, \pi]$ for $j=2, \ldots, N-1$. If $N \geq 4$

$$
\begin{aligned}
& \int_{\mathbb{R}^{N}}\left|x_{1}\right| \mu_{1}(\|x\|) \mathrm{d} x \\
= & \int_{\mathbb{R}_{+}} \int_{0}^{2 \pi} \int_{0}^{\pi} \cdots \int_{0}^{\pi}\left|\cos \theta_{N-1}\right| \rho^{N} \mu_{1}(\rho)\left(\prod_{j=2}^{N-1}\left(\sin \theta_{j}\right)^{j-1}\right) \mathrm{d} \theta_{N-1} \mathrm{~d} \theta_{N-2} \cdots \mathrm{d} \theta_{1} \mathrm{~d} \rho \\
= & \int_{0}^{2 \pi} \int_{0}^{\pi} \cdots \int_{0}^{\pi}\left(\prod_{j=2}^{N-2}\left(\sin \theta_{j}\right)^{j-1}\right) \mathrm{d} \theta_{N-2} \cdots \mathrm{d} \theta_{1} \\
= & (N-1) \omega_{N-1}^{\pi} \frac{2}{N-1} \frac{1}{N \omega_{N}} \int_{\mathbb{R}^{N}}\|x\| \mu_{1}(\|x\|) \mathrm{d} x \\
= & \frac{2}{N} \frac{\omega_{N-1}}{\omega_{N}} \int_{\mathbb{R}^{N}}\|x\| \mu_{1}(\|x\|) \mathrm{d} x
\end{aligned}
$$

completing the proof.

Recall the following theorem (see [1], Theorem 3.9 and Remark 3.10]):

Theorem 4.2 Let $u \in \mathbf{L}_{\text {loc }}^{1}\left(\mathbb{R}^{N} ; \mathbb{R}\right)$, then $u \in \mathbf{B V}\left(\mathbb{R}^{N} ; \mathbb{R}\right)$ if and only if there exists a sequence $u_{n}$ in $\mathbf{C}^{\infty}\left(\mathbb{R}^{N} ; \mathbb{R}\right)$ converging to $u$ in $\mathbf{L}_{\text {loc }}^{\mathbf{1}}$ and satisfying

$$
\lim _{n \rightarrow+\infty} \int_{\mathbb{R}^{N}}\left\|\nabla u_{n}(x)\right\| \mathrm{d} x=L \quad \text { with } \quad L<\infty .
$$

Moreover, $\mathrm{TV}(u)$ is the least constant $L$ for which there exists a sequence as above.

Proposition 4.3 Fix $\mu_{1}$ as in $($ 4.1. $)$. Let $u \in \mathbf{L}_{\text {loc }}^{1}\left(\mathbb{R}^{N} ; \mathbb{R}\right)$ admit a constant $\tilde{C}$ such that for all positive $\lambda, R$ and with $\mu$ as in (4.2)

$$
\frac{1}{\lambda} \int_{\mathbb{R}^{N}} \int_{B\left(x_{o}, R\right)}|u(x)-u(x-z)| \mu(z) \mathrm{d} x \mathrm{~d} z \leq \tilde{C} .
$$

Then, $u \in \mathbf{B V}\left(\mathbb{R}^{N} ; \mathbb{R}\right)$ and $\operatorname{TV}(u) \leq \tilde{C} / C_{1}$, where

$$
C_{1}=\int_{\mathbb{R}^{N}}\left|x_{1}\right| \mu_{1}(\|x\|) \mathrm{d} x .
$$

Note that $\left.C_{1} \in\right] 0,1\left[\right.$. If moreover $u \in \mathbf{C}^{\mathbf{1}}\left(\mathbb{R}^{N} ; \mathbb{R}\right)$, then

$$
\operatorname{TV}(u)=\frac{1}{C_{1}} \lim _{\lambda \rightarrow 0} \frac{1}{\lambda} \int_{\mathbb{R}^{N}} \int_{\mathbb{R}^{N}}|u(x)-u(x-z)| \mu(z) \mathrm{d} x \mathrm{~d} z .
$$


Proof. We introduce now a regularisation of $u$ : $u_{h}=u * \mu_{h}$, with $\mu_{h}(x)=\mu_{1}(\|x\| / h) / h^{N}$. Note that $u_{h} \in \mathbf{C}^{\infty}\left(\mathbb{R}^{N} ; \mathbb{R}\right)$ and $u_{h}$ converges to $u$ in $\mathbf{L}_{\text {loc }}^{1}$ as $h \rightarrow 0$. Furthermore, for $R$ and $h$ positive, we have

$$
\begin{aligned}
& \frac{1}{\lambda} \int_{\mathbb{R}^{N}} \int_{B\left(x_{o}, R\right)}\left|u_{h}(x)-u_{h}(x-z)\right| \mu(z) \mathrm{d} x \mathrm{~d} z \\
\leq & \frac{1}{\lambda} \int_{\mathbb{R}^{N}} \int_{B\left(x_{o}, R+h\right)}|u(x)-u(x-z)| \mu(z) \mathrm{d} x \mathrm{~d} z \\
\leq & \tilde{C}
\end{aligned}
$$

and

$$
\frac{u_{h}(x)-u_{h}(x-\lambda z)}{\lambda}=\int_{0}^{1} \nabla u_{h}(x-\lambda s z) \cdot z \mathrm{~d} s .
$$

Thanks to the Dominated Convergence Theorem, at the limit $\lambda \rightarrow 0$ we get

$$
\int_{\mathbb{R}^{N}} \int_{B\left(x_{o}, R\right)}\left|\nabla u_{h}(x) \cdot z\right| \mu_{1}(\|z\|) \mathrm{d} x \mathrm{~d} z \leq \tilde{C} .
$$

Remark that for fixed $x \in B\left(x_{o}, R\right)$, when $\nabla u_{h}(x) \neq 0$, the scalar product $\nabla u_{h}(x) \cdot z$ is positive (respectively, negative) when $z$ is in a half-space, say $H_{x}^{+}$(respectively, $H_{x}^{-}$). We can write $z=\alpha \frac{\nabla u_{h}(x)}{\left\|\nabla u_{h}(x)\right\|}+w$, with $\alpha \in \mathbb{R}$ and $w$ in the hyperplane $H_{x}^{o}=\nabla u_{h}(x)^{\perp}$. Hence

$$
\begin{aligned}
\int_{\mathbb{R}^{N}}\left|\nabla u_{h}(x) \cdot z\right| \mu_{1}(\|z\|) \mathrm{d} z & =\int_{H_{x}^{+}} \nabla u_{h}(x) \cdot z \mu_{1}(\|z\|) \mathrm{d} z+\int_{H_{x}^{-}} \nabla u_{h}(x) \cdot(-z) \mu_{1}(\|z\|) \mathrm{d} z \\
& =2 \int_{H_{x}^{+}} \nabla u_{h}(x) \cdot z \mu_{1}(\|z\|) \mathrm{d} z \\
& =2 \int_{\mathbb{R}_{+}} \int_{H_{x}^{o}} \alpha\left\|\nabla u_{h}(x)\right\| \mu_{1}\left(\sqrt{\alpha^{2}+\|w\|^{2}}\right) \mathrm{d} w \mathrm{~d} \alpha \\
& =\int_{\mathbb{R}} \int_{H_{x}^{o}}|\alpha|\left\|\nabla u_{h}(x)\right\| \mu_{1}\left(\sqrt{\alpha^{2}+\|w\|^{2}}\right) \mathrm{d} w \mathrm{~d} \alpha \\
& =\left\|\nabla u_{h}(x)\right\| \int_{\mathbb{R}^{N}}\left|z_{1}\right| \mu_{1}(\|z\|) \mathrm{d} z .
\end{aligned}
$$

Define $C_{1}$ as in (4.8) and note that $\left.C_{1} \in\right] 0,1[$. Then we obtain, for all $R>0$,

$$
\int_{B\left(x_{o}, R\right)}\left\|\nabla u_{h}(x)\right\| \mathrm{d} x \leq \frac{\tilde{C}}{C_{1}} .
$$

Finally when $R \rightarrow \infty$ we get $\int_{\mathbb{R}^{N}}\left\|\nabla u_{h}(x)\right\| \mathrm{d} x \leq \tilde{C} / C_{1}$ and in the limit $h \rightarrow 0$, by Theorem 4.2 also $\operatorname{TV}(u) \leq \tilde{C} / C_{1}$, concluding the proof of the first statement.

Assume now that $u \in \mathbf{C}^{\mathbf{1}}\left(\mathbb{R}^{N} ; \mathbb{R}\right)$. Then, using the same computations as above,

$$
\begin{aligned}
& \lim _{\lambda \rightarrow 0} \frac{1}{\lambda} \int_{\mathbb{R}^{N}} \int_{\mathbb{R}^{N}}|u(x)-u(x-z)| \mu(z) \mathrm{d} x \mathrm{~d} z \\
= & \lim _{\lambda \rightarrow 0} \int_{\mathbb{R}^{N}} \int_{\mathbb{R}^{N}}\left|\int_{0}^{1} \nabla u(x-\lambda s z) \cdot z \mathrm{~d} s\right| \mu_{1}(\|z\|) \mathrm{d} x \mathrm{~d} z \\
= & C_{1} \operatorname{TV}(u),
\end{aligned}
$$

completing the proof. 
In the following proof, this property of any function $u \in \mathbf{B V}\left(\mathbb{R}^{N} ; \mathbb{R}\right)$ will be of use:

$$
\int_{\mathbb{R}^{N}}|u(x)-u(x-z)| \mathrm{d} x \leq\|z\| \operatorname{TV}(u) \quad \text { for all } z \in \mathbb{R}^{N} .
$$

For a proof, see [1, Remark 3.25].

Proof of Theorem 2.5. Assume first that $u_{o} \in \mathbf{C}^{\mathbf{1}}\left(\mathbb{R}^{N} ; \mathbb{R}\right)$, the general case will be considered only at the end of this proof.

Let $u$ be the weak entropy solution to (1.1). Denote $u=u(t, x)$ and $v=u(s, y)$ for $(t, x),(s, y) \in \mathbb{R}_{+} \times \mathbb{R}^{N}$. Then, for all $k, l \in \mathbb{R}$ and for all test functions $\varphi=\varphi(t, x, s, y)$ in $\mathbf{C}_{\mathbf{c}}^{\mathbf{1}}\left(\left(\mathbb{R}_{+} \times \mathbb{R}^{N}\right)^{2} ; \overline{\mathbb{R}}_{+}\right)$, we have

$$
\begin{array}{r}
\int_{\mathbb{R}_{+}} \int_{\mathbb{R}^{N}}\left[(u-k) \partial_{t} \varphi+(f(t, x, u)-f(t, x, k)) \nabla_{x} \varphi+(F(t, x, u)-\operatorname{div} f(t, x, k)) \varphi\right] \\
\times \operatorname{sign}(u-k) \mathrm{d} x \mathrm{~d} t \geq 0
\end{array}
$$

for all $(s, y) \in \mathbb{R}_{+} \times \mathbb{R}^{N}$, and

$$
\begin{array}{r}
\int_{\mathbb{R}_{+}} \int_{\mathbb{R}^{N}}\left[(v-l) \partial_{s} \varphi+(f(s, y, v)-f(s, y, l)) \nabla_{y} \varphi+(F(s, y, v)-\operatorname{div} f(s, y, l)) \varphi\right] \\
\times \operatorname{sign}(v-l) \mathrm{d} y \mathrm{~d} s \geq 0
\end{array}
$$

for all $(t, x) \in \mathbb{R}_{+} \times \mathbb{R}^{N}$. Let $\Phi \in \mathbf{C}_{\mathbf{c}}^{\infty}\left(\mathbb{R}_{+} \times \mathbb{R}^{N} ; \overline{\mathbb{R}}_{+}\right), \Psi \in \mathbf{C}_{\mathbf{c}}^{\infty}\left(\mathbb{R} \times \mathbb{R}^{N} ; \overline{\mathbb{R}}_{+}\right)$and set

$$
\varphi(t, x, s, y)=\Phi(t, x) \Psi(t-s, x-y) \text {. }
$$

Observe that $\partial_{t} \varphi+\partial_{s} \varphi=\Psi \partial_{t} \Phi, \nabla_{x} \varphi=\Psi \nabla_{x} \Phi+\Phi \nabla_{x} \Psi, \nabla_{y} \varphi=-\Phi \nabla_{x} \Psi$. Choose $k=v(s, y)$ in (4.12) and integrate with respect to $(s, y)$. Analogously, take $l=u(t, x)$ in $(4.13)$ and integrate with respect to $(t, x)$. Summing the obtained inequalities, we get

$$
\begin{array}{r}
\int_{\mathbb{R}_{+}} \int_{\mathbb{R}^{N}} \int_{\mathbb{R}_{+}} \int_{\mathbb{R}^{N}} \operatorname{sign}(u-v)\left[(u-v) \Psi \partial_{t} \Phi+(f(t, x, u)-f(t, x, v)) \cdot(\nabla \Phi) \Psi\right. \\
+(f(s, y, v)-f(s, y, u)-f(t, x, v)+f(t, x, u)) \cdot(\nabla \Psi) \Phi \\
+(F(t, x, u)-F(s, y, v)+\operatorname{div} f(s, y, u)-\operatorname{div} f(t, x, v)) \varphi] \mathrm{d} x \mathrm{~d} t \mathrm{~d} y \mathrm{~d} s \geq 0 .
\end{array}
$$

Introduce a family of functions $\left\{Y_{\vartheta}\right\}_{\vartheta>0}$ such that for any $\vartheta>0$ :

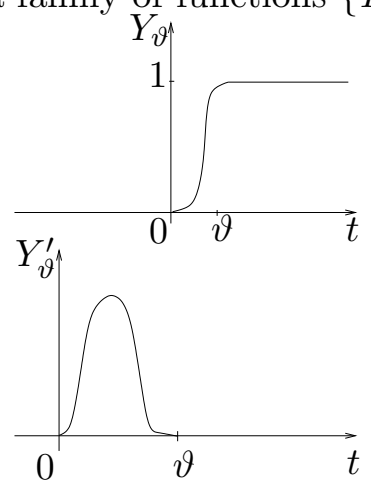

$$
\begin{aligned}
Y_{\vartheta}(t) & =\int_{-\infty}^{t} Y_{\vartheta}^{\prime}(s) \mathrm{d} s \\
Y_{\vartheta}^{\prime}(t) & =\frac{1}{\vartheta} Y^{\prime}\left(\frac{t}{\vartheta}\right) \\
Y^{\prime} & \in \mathbf{C}_{\mathbf{c}}^{\infty}(\mathbb{R} ; \mathbb{R}) \\
\operatorname{supp}\left(Y^{\prime}\right) & \subset] 0,1[ \\
Y^{\prime} & \geq 0 \\
\int_{\mathbb{R}} Y^{\prime}(s) \mathrm{d} s & =1 .
\end{aligned}
$$

Let $M=\left\|\partial_{u} f\right\|_{\mathbf{L}^{\infty}\left(\Omega ; \mathbb{R}^{N}\right)}$ and define for $\varepsilon, \theta, T_{o}, R>0, x_{o} \in \mathbb{R}^{N}$, (see Figure 1):

$$
\chi(t)=Y_{\varepsilon}(t)-Y_{\varepsilon}(t-T) \quad \text { and } \quad \psi(t, x)=1-Y_{\theta}\left(\left\|x-x_{o}\right\|-R-M\left(T_{o}-t\right)\right) \geq 0,
$$




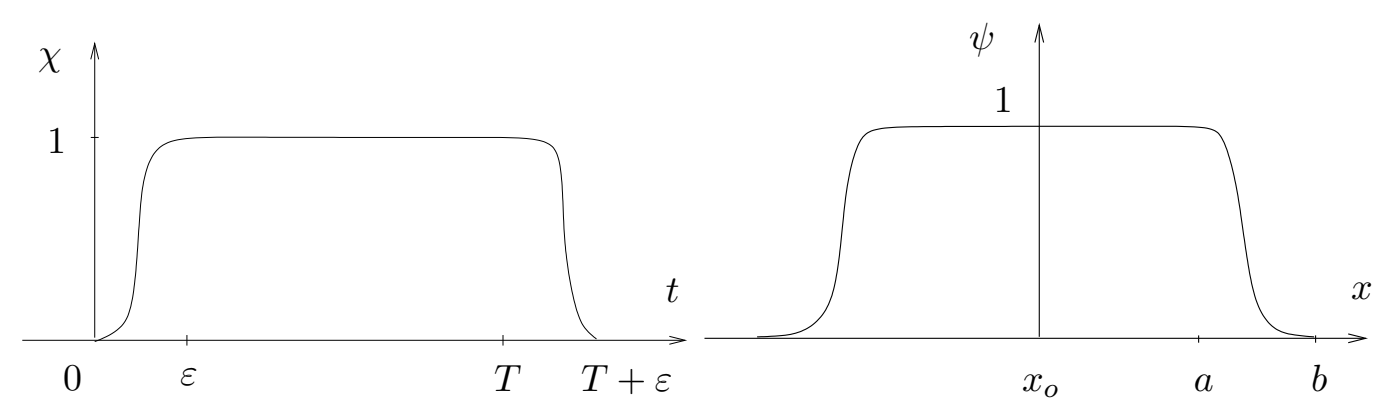

Figure 1: Graphs of $\chi$, left, and of $\psi$, right. Here $a=R+M\left(T_{o}-t\right)$ and $b=R+M\left(T_{o}-t\right)+\theta$.

where we also need the compatibility conditions $T_{o} \geq T$ and $M \varepsilon \leq R+M\left(T_{o}-T\right)$. Observe that $\chi \rightarrow \mathbf{1}_{[0, T]}$ and $\chi^{\prime} \rightarrow \delta_{0}-\delta_{T}$ as $\varepsilon$ tends to 0 . On $\chi$ and $\psi$ we use the bounds

$$
\chi \leq \mathbf{1}_{[0, T+\varepsilon]} \quad \text { and } \quad \mathbf{1}_{B\left(x_{o}, R+M\left(T_{o}-t\right)\right)} \leq \psi \leq \mathbf{1}_{B\left(x_{o}, R+M\left(T_{o}-t\right)+\theta\right)} .
$$

In (4.15), choose $\Phi(t, x)=\chi(t) \psi(t, x)$. With this choice, we have

$$
\partial_{t} \Phi=\chi^{\prime} \psi-M \chi Y_{\theta}^{\prime} \quad \text { and } \quad \nabla \Phi=-\chi Y_{\theta}^{\prime} \frac{x-x_{o}}{\left\|x-x_{o}\right\|} .
$$

Setting $B(t, x, u, v)=|u-v| M+\operatorname{sign}(u-v)(f(t, x, u)-f(t, x, v)) \cdot \frac{x-x_{o}}{\left\|x-x_{o}\right\|}$, the first line in (4.15) becomes

$$
\begin{aligned}
& \int_{\mathbb{R}_{+}} \int_{\mathbb{R}^{N}} \int_{\mathbb{R}_{+}} \int_{\mathbb{R}^{N}}\left[(u-v) \Psi \partial_{t} \Phi+(f(t, x, u)-f(t, x, v))(\nabla \Phi) \Psi\right] \operatorname{sign}(u-v) \mathrm{d} x \mathrm{~d} t \mathrm{~d} y \mathrm{~d} s \\
= & \int_{\mathbb{R}_{+}} \int_{\mathbb{R}^{N}} \int_{\mathbb{R}_{+}} \int_{\mathbb{R}^{N}}\left(|u-v| \chi^{\prime} \psi-B(t, x, u, v) \chi Y_{\theta}^{\prime}\right) \Psi \mathrm{d} x \mathrm{~d} t \mathrm{~d} y \mathrm{~d} s \\
\leq & \int_{\mathbb{R}_{+}} \int_{\mathbb{R}^{N}} \int_{\mathbb{R}_{+}} \int_{\mathbb{R}^{N}}|u-v| \chi^{\prime} \psi \Psi \mathrm{d} x \mathrm{~d} t \mathrm{~d} y \mathrm{~d} s
\end{aligned}
$$

since $B(t, x, u, v)$ is positive for all $(t, x, u, v) \in \Omega \times \mathbb{R}$. Thanks to the above estimate and to $(4.15)$, we have

$$
\begin{aligned}
\int_{\mathbb{R}_{+}} \int_{\mathbb{R}^{N}} \int_{\mathbb{R}_{+}} \int_{\mathbb{R}^{N}} & {\left[(u-v) \chi^{\prime} \psi \Psi\right.} \\
& +(f(s, y, v)-f(s, y, u)-f(t, x, v)+f(t, x, u)) \cdot(\nabla \Psi) \Phi \\
& +(F(t, x, u)-F(s, y, v)-\operatorname{div} f(t, x, v)+\operatorname{div} f(s, y, u)) \varphi] \geq 0 . \\
& \times \operatorname{sign}(u-v) \mathrm{d} x \mathrm{~d} t \mathrm{~d} y \mathrm{~d} s \quad \geq 0
\end{aligned}
$$

Now, we aim at bounds for each term of this sum. Introduce the following notations:

$$
\begin{gathered}
I=\int_{\mathbb{R}_{+}} \int_{\mathbb{R}^{N}} \int_{\mathbb{R}_{+}} \int_{\mathbb{R}^{N}}|u-v| \chi^{\prime} \psi \Psi \mathrm{d} x \mathrm{~d} t \mathrm{~d} y \mathrm{~d} s, \\
J_{x}=\int_{\mathbb{R}_{+}} \int_{\mathbb{R}^{N}} \int_{\mathbb{R}_{+}} \int_{\mathbb{R}^{N}}(f(t, y, v)-f(t, y, u)+f(t, x, u)-f(t, x, v))(\nabla \Psi) \Phi \\
\times \operatorname{sign}(u-v) \mathrm{d} x \mathrm{~d} t \mathrm{~d} y \mathrm{~d} s,
\end{gathered}
$$




$$
\begin{aligned}
J_{t}=\int_{\mathbb{R}_{+}} \int_{\mathbb{R}^{N}} \int_{\mathbb{R}_{+}} \int_{\mathbb{R}^{N}}(f(s, y, v)-f(s, y, u)+f(t, y, u)-f(t, y, v))(\nabla \Psi) \Phi \\
\quad \times \operatorname{sign}(u-v) \mathrm{d} x \mathrm{~d} t \mathrm{~d} y \mathrm{~d} s, \\
L_{x}=\int_{\mathbb{R}_{+}} \int_{\mathbb{R}^{N}} \int_{\mathbb{R}_{+}} \int_{\mathbb{R}^{N}}(F(t, x, u)-F(t, y, v)-\operatorname{div} f(t, x, v)+\operatorname{div} f(t, y, u)) \varphi \\
\quad \times \operatorname{sign}(u-v) \mathrm{d} x \mathrm{~d} t \mathrm{~d} y \mathrm{~d} s, \\
L_{t}=\int_{\mathbb{R}_{+}} \int_{\mathbb{R}^{N}} \int_{\mathbb{R}_{+}} \int_{\mathbb{R}^{N}}(F(t, y, v)-F(s, y, v)-\operatorname{div} f(t, y, u)+\operatorname{div} f(s, y, u)) \varphi \\
\times \operatorname{sign}(u-v) \mathrm{d} x \mathrm{~d} t \mathrm{~d} y \mathrm{~d} s .
\end{aligned}
$$

Then, the above inequality is rewritten as $I+J_{x}+J_{t}+L_{x}+L_{t} \geq 0$. Choose $\Psi(t, x)=$ $\nu(t) \mu(x)$ where, for $\eta, \lambda>0, \mu \in \mathbf{C}_{\mathbf{c}}^{\infty}\left(\overline{\mathbb{R}}_{+} ; \overline{\mathbb{R}}_{+}\right)$satisfies (4.1)-(4.2) and

$$
\left.\nu(t)=\frac{1}{\eta} \nu_{1}\left(\frac{t}{\eta}\right), \quad \int_{\mathbb{R}} \nu_{1}(s) \mathrm{d} s=1, \quad \nu_{1} \in \mathbf{C}_{\mathbf{c}}^{\infty}\left(\mathbb{R} ; \overline{\mathbb{R}}_{+}\right), \quad \operatorname{supp}\left(\nu_{1}\right) \subset\right]-1,0[.
$$

We have

$$
\begin{aligned}
I & \leq I_{1}+I_{2} \text { where } \\
I_{1} & =\int_{\mathbb{R}_{+}} \int_{\mathbb{R}^{N}} \int_{\mathbb{R}_{+}} \int_{\mathbb{R}^{N}}|u(t, x)-u(t, y)|\left(Y_{\varepsilon}^{\prime}(t)-Y_{\varepsilon}^{\prime}(t-T)\right) \psi \Psi \mathrm{d} x \mathrm{~d} t \mathrm{~d} y \mathrm{~d} s, \\
I_{2} & =\int_{\mathbb{R}_{+}} \int_{\mathbb{R}^{N}} \int_{\mathbb{R}_{+}} \int_{\mathbb{R}^{N}}|u(t, y)-u(s, y)|\left(Y_{\varepsilon}^{\prime}(t)+Y_{\varepsilon}^{\prime}(t-T)\right) \psi \Psi \mathrm{d} x \mathrm{~d} t \mathrm{~d} y \mathrm{~d} s
\end{aligned}
$$

and we get

$$
\begin{aligned}
\limsup _{\varepsilon \rightarrow 0} I_{1} \leq & \int_{\mathbb{R}^{N}} \int_{\left\|x-x_{o}\right\| \leq R+M T_{o}+\theta}|u(0, x)-u(0, y)| \mu(x-y) \mathrm{d} x \mathrm{~d} y \\
& -\int_{\mathbb{R}^{N}} \int_{\left\|x-x_{o}\right\| \leq R+M\left(T_{o}-T\right)}|u(T, x)-u(T, y)| \mu(x-y) \mathrm{d} x \mathrm{~d} y, \\
\limsup _{\varepsilon \rightarrow 0} I_{2} \leq & 2 \sup _{\substack{t \in\{0, T\}, s \in t t, t+\eta[}} \int_{\left\|y-x_{o}\right\| \leq R+\lambda+M\left(T_{o}-t\right)+\theta}|u(t, y)-u(s, y)| \mathrm{d} y .
\end{aligned}
$$

For $J_{x}$, we have that by (H1), $f \in \mathbf{C}^{2}\left(\Omega ; \mathbb{R}^{N}\right)$ and therefore

$$
\begin{aligned}
& \|f(t, y, v)-f(t, y, u)+f(t, x, u)-f(t, x, v)\|= \\
= & \left\|\int_{u}^{v} \int_{0}^{1} \nabla \partial_{u} f(t, x(1-r)+r y, w) \cdot(y-x) \mathrm{d} r \mathrm{~d} w\right\| \\
\leq & \left\|\nabla \partial_{u} f\right\|_{\mathbf{L}^{\infty}\left(\Omega ; \mathbb{R}^{N \times N)}\right)}\|x-y\||u(s, y)-u(t, x)| .
\end{aligned}
$$

Then, using (4.5)

$$
\begin{aligned}
J_{x} \leq\left\|\nabla \partial_{u} f\right\|_{\mathbf{L}^{\infty}} \int_{\mathbb{R}_{+}} \int_{\mathbb{R}^{N}} \int_{\mathbb{R}_{+}} \int_{\mathbb{R}^{N}}\|x-y\||u(t, x)-u(s, y)|\|\nabla \Psi\| \chi \psi \mathrm{d} x \mathrm{~d} t \mathrm{~d} y \mathrm{~d} s \\
\leq\left\|\nabla \partial_{u} f\right\|_{\mathbf{L}^{\infty}} \int_{\mathbb{R}_{+}} \int_{\mathbb{R}^{N}} \int_{\mathbb{R}_{+}} \int_{\mathbb{R}^{N}}\|x-y\|[|u(t, y)-u(s, y)|+|u(t, x)-u(t, y)|] \\
\times\|\nabla \Psi\| \chi \psi \mathrm{d} x \mathrm{~d} t \mathrm{~d} y \mathrm{~d} s
\end{aligned}
$$




$$
\begin{aligned}
\leq & N\left\|\nabla \partial_{u} f\right\|_{\mathbf{L}^{\infty}}(T+\varepsilon) \sup _{\substack{t \in[0, T+\varepsilon], s \in], t+\eta]}} \int_{\left\|y-x_{o}\right\| \leq R+\lambda+M\left(T_{o}-t\right)+\theta}|u(t, y)-u(s, y)| \mathrm{d} y \\
& +\left\|\nabla \partial_{u} f\right\|_{\mathbf{L}^{\infty}} \int_{0}^{T+\varepsilon} \int_{\mathbb{R}^{N}} \int_{B\left(x_{o}, R+M\left(T_{o}-t\right)+\theta\right)}\|x-y\||u(t, x)-u(t, y)|\|\nabla \mu\| \mathrm{d} x \mathrm{~d} y \mathrm{~d} t, \\
J_{t} \leq & \int_{\mathbb{R}_{+}} \int_{\mathbb{R}^{N}} \int_{\mathbb{R}_{+}} \int_{\mathbb{R}^{N}}\left\|\int_{s}^{t} \int_{v}^{u} \partial_{t} \partial_{u} f(\tau, y, w) \mathrm{d} w \mathrm{~d} \tau\right\|\|\nabla \Psi\| \Phi \mathrm{d} x \mathrm{~d} t \mathrm{~d} y \mathrm{~d} s \\
\leq & \eta\left\|\partial_{t} \partial_{u} f\right\|_{\mathbf{L}^{\infty}} \int_{\mathbb{R}_{+}} \int_{\mathbb{R}^{N}} \int_{\mathbb{R}_{+}} \int_{\mathbb{R}^{N}}|u(t, x)-u(s, y)|\|\nabla \Psi\| \Phi \mathrm{d} x \mathrm{~d} t \mathrm{~d} y \mathrm{~d} s .
\end{aligned}
$$

For $L_{x}$, we get

$$
\begin{aligned}
& L_{x}= L_{1}+L_{2} \\
& L_{1}=\int_{\mathbb{R}_{+}} \int_{\mathbb{R}^{N}} \int_{\mathbb{R}_{+}} \int_{\mathbb{R}^{N}}\left[\int_{v}^{u}\left(\partial_{u} \operatorname{div} f(t, x, w)+\partial_{u} F(t, y, w)\right) \mathrm{d} w\right] \varphi \operatorname{sign}(u-v) \mathrm{d} x \mathrm{~d} t \mathrm{~d} y \mathrm{~d} s, \\
& L_{2}=\int_{\mathbb{R}_{+}} \int_{\mathbb{R}^{N}} \int_{\mathbb{R}_{+}} \int_{\mathbb{R}^{N}}\left[\int_{0}^{1} \nabla(F-\operatorname{div} f)(t, r x+(1-r) y, u) \cdot(x-y) \mathrm{d} r\right] \varphi \\
& \times \operatorname{sign}(u-v) \mathrm{d} x \mathrm{~d} t \mathrm{~d} y \mathrm{~d} s .
\end{aligned}
$$

Then, recalling (4.14), the definitions $\Psi=\nu \mu, \Phi=\chi \psi,(4.1)$, (4.19) and (4.17), we obtain

$$
\begin{aligned}
L_{1} \leq & \left(N\left\|\nabla \partial_{u} f\right\|_{\mathbf{L}^{\infty}}+\left\|\partial_{u} F\right\|_{\mathbf{L}^{\infty}}\right) \\
& \times\left[(T+\varepsilon) \sup _{\substack{t \in[0, T+\varepsilon], s \in t, t+\eta]}} \int_{\left\|y-x_{o}\right\| \leq R+\lambda+M\left(T_{o}-t\right)+\theta}|u(t, y)-u(s, y)| \mathrm{d} y\right. \\
& \left.\quad+\int_{0}^{T+\varepsilon} \int_{\mathbb{R}^{N}} \int_{\left\|x-x_{o}\right\| \leq R+M\left(T_{o}-t\right)+\theta}|u(t, x)-u(t, y)| \mu(x-y) \mathrm{d} x \mathrm{~d} y \mathrm{~d} t\right], \\
L_{2} \leq & \int_{\mathbb{R}_{+}} \int_{\mathbb{R}^{N}} \int_{\mathbb{R}_{+}} \int_{\mathbb{R}^{N}} \int_{0}^{1}\|\nabla(F-\operatorname{div} f)(t, y+r(x-y), u)\|\|x-y\| \chi \psi \mu \nu \mathrm{d} r \mathrm{~d} x \mathrm{~d} t \mathrm{~d} y \mathrm{~d} s \\
\leq & \left(\int_{0}^{T+\varepsilon} \int_{\mathbb{R}^{N}}\|\nabla(F-\operatorname{div} f)(t, y, \cdot)\|_{\mathbf{L}^{\infty}} \mathrm{d} y \mathrm{~d} t\right) \int_{\mathbb{R}^{N}}\|x\| \mu(x) \mathrm{d} x \\
= & \lambda M_{1} \int_{0}^{T+\varepsilon} \int_{\mathbb{R}^{N}}\|\nabla(F-\operatorname{div} f)(t, y, \cdot)\|_{\mathbf{L}^{\infty}} \mathrm{d} y \mathrm{~d} t
\end{aligned}
$$

where

$$
M_{1}=\int_{\mathbb{R}^{N}}\|x\| \mu_{1}(\|x\|) \mathrm{d} x .
$$

Concerning the latter term $L_{t}$

$$
L_{t} \leq \eta \omega_{N}\left(R+M T_{o}\right)^{N}(T+\varepsilon)\left(\left\|\partial_{t} \operatorname{div} f\right\|_{\mathbf{L}^{\infty}}+\left\|\partial_{t} F\right\|_{\mathbf{L}^{\infty}}\right) .
$$

Letting $\varepsilon, \eta, \theta \rightarrow 0$ we get

$$
\begin{aligned}
\limsup _{\varepsilon, \eta, \theta \rightarrow 0} I_{1}= & \int_{\mathbb{R}^{N}} \int_{\left\|x-x_{o}\right\| \leq R+M T_{o}}|u(0, x)-u(0, y)| \mu(x-y) \mathrm{d} x \mathrm{~d} y \\
& -\int_{\mathbb{R}^{N}} \int_{\left\|x-x_{o}\right\| \leq R+M\left(T_{o}-T\right)}|u(T, x)-u(T, y)| \mu(x-y) \mathrm{d} x \mathrm{~d} y,
\end{aligned}
$$




$$
\begin{aligned}
& \limsup _{\varepsilon, \eta, \theta \rightarrow 0} I_{2}=0, \\
& \limsup _{\varepsilon, \eta, \theta \rightarrow 0} J_{x} \leq\left\|\nabla \partial_{u} f\right\|_{\mathbf{L}^{\infty}} \int_{0}^{T} \int_{\mathbb{R}^{N}} \int_{B\left(x_{o}, R+M\left(T_{o}-t\right)\right)}\|x-y\||u(t, x)-u(t, y)| \\
& \limsup _{\varepsilon, \eta, \theta \rightarrow 0} J_{t}=0, \quad \times\|\nabla \mu(x-y)\| \mathrm{d} x \mathrm{~d} y \mathrm{~d} t, \\
& \limsup _{\varepsilon, \eta, \theta \rightarrow 0} L_{1} \leq \quad\left(N\left\|\nabla \partial_{u} f\right\|_{\mathbf{L}^{\infty}}+\left\|\partial_{u} F\right\|_{\mathbf{L}^{\infty}}\right) \\
& \quad \times \int_{0}^{T} \int_{\mathbb{R}^{N}} \int_{\left\|x-x_{o}\right\| \leq R+M\left(T_{o}-t\right)}|u(t, x)-u(t, y)| \mu(x-y) \mathrm{d} x \mathrm{~d} y \mathrm{~d} t, \\
& \limsup _{\varepsilon, \eta, \theta \rightarrow 0} L_{2} \leq \lambda M_{1} \int_{0}^{T} \int_{\mathbb{R}^{N}}\|\nabla(F-\operatorname{div} f)(t, y, \cdot)\|_{\mathbf{L}^{\infty}} \mathrm{d} y \mathrm{~d} t, \\
& \limsup _{\varepsilon, \eta, \theta \rightarrow 0} L_{t}=0 .
\end{aligned}
$$

Collating all the obtained results and using the equality $\|\nabla \mu(x)\|=-\frac{1}{\lambda^{N+1}} \mu_{1}^{\prime}\left(\frac{\|x\|}{\lambda}\right)$

$$
\begin{gathered}
\int_{\mathbb{R}^{N}} \int_{\left\|x-x_{o}\right\| \leq R+M\left(T_{o}-T\right)}|u(T, x)-u(T, y)| \frac{1}{\lambda^{N}} \mu_{1}\left(\frac{\|x-y\|}{\lambda}\right) \mathrm{d} x \mathrm{~d} y \\
\leq \int_{\mathbb{R}^{N}} \int_{\left\|x-x_{o}\right\| \leq R+M\left(T_{o}-T\right)}|u(0, x)-u(0, y)| \frac{1}{\lambda^{N}} \mu_{1}\left(\frac{\|x-y\|}{\lambda}\right) \mathrm{d} x \mathrm{~d} y \\
-\left\|\nabla \partial_{u} f\right\|_{\mathbf{L}^{\infty}} \int_{0}^{T} \int_{\mathbb{R}^{N}} \int_{\left\|x-x_{o}\right\| \leq R+M\left(T_{o}-t\right)}|u(t, x)-u(t, y)| \\
\times \frac{1}{\lambda^{N+1}} \mu_{1}^{\prime}\left(\frac{\|x-y\|}{\lambda}\right)\|x-y\| \mathrm{d} x \mathrm{~d} y \mathrm{~d} t \\
+\left(N\left\|\nabla \partial_{u} f\right\|_{\mathbf{L}^{\infty}}+\left\|\partial_{u} F\right\|_{\left.\mathbf{L}^{\infty}\right) \int_{0}^{T}} \int_{\mathbb{R}^{N}} \int_{\left\|x-x_{o}\right\| \leq R+M\left(T_{o}-t\right)}|u(t, x)-u(t, y)|\right. \\
\times \frac{1}{\lambda^{N}} \mu_{1}\left(\frac{\|x-y\|}{\lambda}\right) \mathrm{d} x \mathrm{~d} y \mathrm{~d} t \\
+\lambda M_{1} \int_{0}^{T} \int_{\mathbb{R}^{N}}\|\nabla(F-\operatorname{div} f)(t, y, \cdot)\|_{\mathbf{L}^{\infty}} \mathrm{d} y \mathrm{~d} t .
\end{gathered}
$$

If $\left\|\nabla \partial_{u} f\right\|_{\mathbf{L}^{\infty}}=\left\|\partial_{u} F\right\|_{\mathbf{L}^{\infty}}=0$ and under the present assumption that $u_{o} \in \mathbf{C}^{\mathbf{1}}\left(\mathbb{R}^{N} ; \mathbb{R}\right)$, using Proposition 4.3, (4.8) and 4.20), we directly obtain that

$$
\operatorname{TV}(u(T)) \leq \operatorname{TV}\left(u_{o}\right)+\frac{M_{1}}{C_{1}} \int_{0}^{T} \int_{\mathbb{R}^{N}}\|\nabla(F-\operatorname{div} f)(t, y, \cdot)\|_{\mathbf{L}^{\infty}} \mathrm{d} y \mathrm{~d} t .
$$

The same procedure at the end of this proof allows to extend (4.22) to more general initial data, providing an estimate of TV $(u(t))$ in the situation studied in [3].

Now, it remains to treat the case $\left\|\nabla \partial_{u} f\right\|_{\mathbf{L}^{\infty}} \neq 0$. A direct use of Gronwall type inequalities is apparently impossible, due to the term with $\nabla \mu$. However, introduce the function

$$
\mathcal{F}(T, \lambda)=\int_{0}^{T} \int_{\mathbb{R}^{N}} \int_{\left\|x-x_{o}\right\| \leq R+M\left(T_{o}-t\right)}|u(t, x)-u(t, x-z)| \frac{1}{\lambda^{N}} \mu_{1}\left(\frac{\|z\|}{\lambda}\right) \mathrm{d} x \mathrm{~d} z \mathrm{~d} t
$$


so that

$$
\begin{aligned}
\partial_{\lambda} \mathcal{F}= & -\frac{N}{\lambda} \mathcal{F} \\
& -\frac{1}{\lambda} \int_{0}^{T} \int_{\mathbb{R}^{N}} \int_{\left\|x-x_{o}\right\| \leq R+M\left(T_{o}-t\right)}|u(t, x)-u(t, x-z)| \frac{\mu_{1}^{\prime}(\|z\| / \lambda)}{\lambda^{N+1}}\|z\| \mathrm{d} x \mathrm{~d} z \mathrm{~d} t .
\end{aligned}
$$

Denote $C(T)=M_{1} \int_{0}^{T} \int_{\mathbb{R}^{N}}\|\nabla(F-\operatorname{div} f)(t, y, \cdot)\|_{\mathbf{L}^{\infty}} \mathrm{d} y \mathrm{~d} t$ and integrate (4.21) on $\left[0, T^{\prime}\right]$ with respect to $T$ for $T^{\prime} \leq T_{o}$. It results

$$
\begin{aligned}
\frac{1}{\lambda} \mathcal{F}\left(T^{\prime}, \lambda\right) \leq & \frac{T^{\prime}}{\lambda} \int_{\mathbb{R}^{N}} \int_{\left\|x-x_{o}\right\| \leq R+M T_{o}}|u(0, x)-u(0, y)| \mu(x-y) \mathrm{d} x \mathrm{~d} y \\
& +T^{\prime}\left\|\nabla \partial_{u} f\right\|_{\mathbf{L}^{\infty}} \partial_{\lambda} \mathcal{F}\left(T^{\prime}, \lambda\right)+\frac{T^{\prime}}{\lambda}\left(2 N\left\|\nabla \partial_{u} f\right\|_{\mathbf{L}^{\infty}}+\left\|\partial_{u} F\right\|_{\mathbf{L}^{\infty}}\right) \mathcal{F}\left(T^{\prime}, \lambda\right) \\
& +T^{\prime} C\left(T^{\prime}\right) .
\end{aligned}
$$

Denote $\alpha=\left(2 N\left\|\nabla \partial_{u} f\right\|_{\mathbf{L}^{\infty}}+\left\|\partial_{u} F\right\|_{\mathbf{L}^{\infty}}-\frac{1}{T^{\prime}}\right)\left(\left\|\nabla \partial_{u} f\right\|_{\mathbf{L}^{\infty}}\right)^{-1}$, so that $\lim _{T^{\prime} \rightarrow 0} \alpha=-\infty$. The previous inequality reads, using (4.11) for $u_{o}$,

$$
\begin{aligned}
\partial_{\lambda} \mathcal{F}\left(T^{\prime}, \lambda\right)+\alpha \frac{\mathcal{F}\left(T^{\prime}, \lambda\right)}{\lambda} & \geq-\left(M_{1} \operatorname{TV}\left(u_{o}\right)+C\left(T^{\prime}\right)\right) \frac{1}{\left\|\nabla \partial_{u} f\right\|_{\mathbf{L}^{\infty}}}, \\
\partial_{\lambda}\left(\lambda^{\alpha} \mathcal{F}\left(T^{\prime}, \lambda\right)\right) & \geq-\lambda^{\alpha}\left(M_{1} \operatorname{TV}\left(u_{o}\right)+C\left(T^{\prime}\right)\right) \frac{1}{\left\|\nabla \partial_{u} f\right\|_{\mathbf{L}^{\infty}}} .
\end{aligned}
$$

Finally, if $T^{\prime}$ is such that $\alpha<-1$, then we integrate in $\lambda$ on $[\lambda,+\infty[$ and we get

$$
\frac{1}{\lambda} \mathcal{F}\left(T^{\prime}, \lambda\right) \leq \frac{1}{-\alpha-1}\left(M_{1} \operatorname{TV}\left(u_{o}\right)+C\left(T^{\prime}\right)\right) \frac{1}{\left\|\nabla \partial_{u} f\right\|_{\mathbf{L}^{\infty}}} .
$$

Furthermore, by (4.1) and (4.2), there exists a constant $K>0$ such that for all $z \in \mathbb{R}^{N}$

$$
-\mu_{1}^{\prime}(\|z\|) \leq K \mu_{1}\left(\frac{\|z\|}{2}\right) .
$$

Divide both sides in (4.21) by $\lambda$, rewrite them using (4.23), (4.24), apply (4.11) and obtain

$$
\begin{aligned}
& \frac{1}{\lambda} \int_{\mathbb{R}^{N}} \int_{\left\|x-x_{o}\right\| \leq R+M\left(T_{o}-T\right)}|u(T, x)-u(T, y)| \frac{1}{\lambda^{N}} \mu_{1}\left(\frac{\|x-y\|}{\lambda}\right) \mathrm{d} x \mathrm{~d} y \\
\leq & M_{1} \operatorname{TV}\left(u_{o}\right)+\frac{\mathcal{F}(T, 2 \lambda)}{2 \lambda} 2^{N+2} K\left\|\nabla \partial_{u} f\right\|_{\mathbf{L}^{\infty}}+\frac{\mathcal{F}(T, \lambda)}{\lambda}\left(2 N\left\|\nabla \partial_{u} f\right\|_{\mathbf{L}^{\infty}}+\left\|\partial_{u} F\right\|_{\mathbf{L}^{\infty}}\right) \\
& +M_{1} \int_{0}^{T} \int_{\mathbb{R}^{N}}\|\nabla(F-\operatorname{div} f)(t, y, \cdot)\|_{\mathbf{L}^{\infty}} \mathrm{d} y \mathrm{~d} t .
\end{aligned}
$$

An application of (4.23) yields an estimate of the type

$$
\frac{1}{\lambda} \int_{\mathbb{R}^{N}} \int_{B\left(x_{o}, R+M\left(T_{o}-T\right)\right)}|u(T, x)-u(T, x-z)| \mu(z) \mathrm{d} x \mathrm{~d} z \leq \check{C},
$$

the positive constant $\check{C}$ being independent from $R$ and $\lambda$. Applying Proposition 4.3 we obtain that $u(t) \in \mathbf{B V}\left(\mathbb{R}^{N} ; \mathbb{R}\right)$ for $t \in\left[0,2 T_{1}[\right.$, where

$$
T_{1}=\frac{1}{2\left((1+2 N)\left\|\nabla \partial_{u} f\right\|_{\mathbf{L}^{\infty}}+\left\|\partial_{u} F\right\|_{\mathbf{L}^{\infty}}\right)} .
$$


The next step is to obtain a general estimate of the TV norm. The starting point is (4.21). Recall the definitions (4.20) of $M_{1}$ and (4.26) of $T_{1}$. Moreover, by (4.6),

$$
\int_{\mathbb{R}^{N}}\|z\|^{2} \mu_{1}^{\prime}(\|z\|) \mathrm{d} z=-(N+1) M_{1} .
$$

Divide both terms in (4.21) by $\lambda$, apply (4.9) on the first term in the right hand side, apply (4.11) on the second and third terms and obtain for all $T \in\left[0, T_{1}\right]$ with $T_{1}<T_{o}$

$$
\begin{aligned}
\operatorname{TV}(u(T)) \leq & \operatorname{TV}\left(u_{o}\right)+\left((2 N+1)\left\|\nabla \partial_{u} f\right\|_{\mathbf{L}^{\infty}}+\left\|\partial_{u} F\right\|_{\mathbf{L}^{\infty}}\right) \frac{M_{1}}{C_{1}} \int_{0}^{T} \operatorname{TV}(u(t)) \mathrm{d} t \\
& +\frac{M_{1}}{C_{1}} \int_{0}^{T} \int_{\mathbb{R}^{N}}\|\nabla(F-\operatorname{div} f)(t, x, \cdot)\|_{\mathbf{L}^{\infty}} \mathrm{d} x \mathrm{~d} t .
\end{aligned}
$$

An application of Gronwall Lemma shows that TV $(u(t))$ is bounded on $\left[0, T_{1}\right]$. Indeed,

$$
\operatorname{TV}(u(t)) \leq e^{\kappa_{o} t} \operatorname{TV}\left(u_{o}\right)+\frac{M_{1}}{C_{1}} \int_{0}^{T} e^{\kappa_{o}(T-t)} \int_{\mathbb{R}^{N}}\|\nabla(F-\operatorname{div} f)(t, x, \cdot)\|_{\mathbf{L}^{\infty}} \mathrm{d} x \mathrm{~d} t
$$

for $t \in\left[0, T_{1}\right], M_{1}, C_{1}$ as in (4.20), (4.8) and $\kappa_{o}=\left[(2 N+1)\left\|\nabla \partial_{u} f\right\|_{\mathbf{L}^{\infty}}+\left\|\partial_{u} F\right\|_{\mathbf{L}^{\infty}}\right] M_{1} / C_{1}$.

We now relax the assumption on the regularity of $u_{o}$. Indeed, let $u_{o} \in \mathbf{B V}\left(\mathbb{R}^{N} ; \mathbb{R}\right)$ and choose a sequence $u_{o}^{n}$ of $\mathbf{C}^{\mathbf{1}}\left(\mathbb{R}^{N} ; \mathbb{R}\right)$ functions such that $\operatorname{TV}\left(u_{o}^{n}\right) \rightarrow \operatorname{TV}\left(u_{o}\right)$, as in Theorem 1.2. Then, by Theorem 2.3, the solutions $u^{n}$ to (1.1) with initial datum $u_{o}^{n}$ satisfy

$$
\lim _{n \rightarrow+\infty} u^{n}(t)=u(t) \text { in } \mathbf{L}_{\mathbf{l o c}}^{\mathbf{1}} \quad \text { and } \quad \mathrm{TV}(u(t)) \leq \liminf _{n \rightarrow+\infty} \operatorname{TV}\left(u^{n}(t)\right),
$$

where we used also the lower semicontinuity of the total variation. Note that (4.27), as well as the relations above, holds for all $t \in\left[0, T_{1}\right], T_{1}$ being independent from the initial datum. Therefore, the bound (4.27) holds for all BV initial data.

Remark that the bound (4.27) is additive in time, in the sense that applying it iteratively for times $T_{1}$ and $t$ yields (4.27) for time $T_{1}+t$ :

$$
\begin{aligned}
& \operatorname{TV}\left(u\left(T_{1}+t\right)\right) \\
\leq & e^{\kappa_{o} t} \operatorname{TV}\left(u\left(T_{1}\right)\right)+\frac{M_{1}}{C_{1}} \int_{T_{1}}^{T_{1}+t} e^{\kappa_{o}(t-s)} \int_{\mathbb{R}^{N}}\|\nabla(F-\operatorname{div} f)(s, x, \cdot)\|_{\mathbf{L}^{\infty}} \mathrm{d} x \mathrm{~d} s \\
\leq & e^{\kappa_{o} t}\left[e^{\kappa_{o} T_{1}} \mathrm{TV}\left(u_{o}\right)+\frac{M_{1}}{C_{1}} \int_{0}^{T_{1}} e^{\kappa_{o}\left(T_{1}-s\right)} \int_{\mathbb{R}^{N}}\|\nabla(F-\operatorname{div} f)(s, x, \cdot)\|_{\mathbf{L}^{\infty}} \mathrm{d} x \mathrm{~d} s\right] \\
& +\frac{M_{1}}{C_{1}} \int_{T_{1}}^{T_{1}+t} e^{\kappa_{o}\left(T_{1}+t-s\right)} \int_{\mathbb{R}^{N}}\|\nabla(F-\operatorname{div} f)(s, x, \cdot)\|_{\mathbf{L}^{\infty}} \mathrm{d} x \mathrm{~d} s \\
= & e^{\kappa_{o}\left(T_{1}+t\right)} \operatorname{TV}\left(u_{o}\right)+\frac{M_{1}}{C_{1}} \int_{0}^{T_{1}+t} e^{\kappa_{o}\left(T_{1}+t-s\right)} \int_{\mathbb{R}^{N}}\|\nabla(F-\operatorname{div} f)(s, x, \cdot)\|_{\mathbf{L}^{\infty}} \mathrm{d} x \mathrm{~d} s .
\end{aligned}
$$

The bound (4.27) can then be applied iteratively, thanks to the fact that $T_{1}$ is independent from the initial datum. An iteration argument allows to prove (2.5) for $t \in\left[0, T_{o}\right]$. The final bound (2.5) then follows by the arbitrariness of $T_{o}$, thanks to (2.1). 


\section{Proof of Theorem 2.6.}

The following proof relies on developing the techniques used in the proof of Theorem 2.5.

Proof of Theorem 2.6. Let $\Phi \in \mathbf{C}_{\mathbf{c}}^{\infty}\left(\mathbb{R}_{+} \times \mathbb{R}^{N} ; \overline{\mathbb{R}}_{+}\right), \Psi \in \mathbf{C}_{\mathbf{c}}^{\infty}\left(\mathbb{R} \times \mathbb{R}^{N} ; \overline{\mathbb{R}}_{+}\right)$and set $\varphi(t, x, s, y)=\Phi(t, x) \Psi(t-s, x-y)$ as in (4.14).

By Definition 2.1, we have $\forall l \in \mathbb{R}, \forall(t, x) \in \mathbb{R}_{+} \times \mathbb{R}^{N}$

$$
\begin{array}{r}
\int_{\mathbb{R}_{+}} \int_{\mathbb{R}^{N}}\left[(u-l) \partial_{s} \varphi+(f(s, y, u)-f(s, y, l)) \cdot \nabla_{y} \varphi+(F(s, y, u)-\operatorname{div} f(s, y, l)) \varphi\right] \\
\times \operatorname{sign}(u-l) \mathrm{d} y \mathrm{~d} s \geq 0
\end{array}
$$

and $\forall k \in \mathbb{R}, \forall(s, y) \in \mathbb{R}_{+} \times \mathbb{R}^{N}$

$$
\begin{array}{r}
\int_{\mathbb{R}_{+}} \int_{\mathbb{R}^{N}}\left[(v-k) \partial_{t} \varphi+(g(t, x, v)-g(t, x, k)) \cdot \nabla_{x} \varphi+(G(t, x, v)-\operatorname{div} g(t, x, k)) \varphi\right] \\
\times \operatorname{sign}(v-k) \mathrm{d} x \mathrm{~d} t \geq 0 .
\end{array}
$$

Choose $k=u(s, y)$ in (5.2) and integrate with respect to $(s, y)$. Analogously, take $l=v(t, x)$ in (5.1) and integrate with respect to $(t, x)$. By summing the obtained equations, we get, denoting $u=u(s, y)$ and $v=v(t, x)$ :

$$
\begin{aligned}
\int_{\mathbb{R}_{+}} \int_{\mathbb{R}^{N}} \int_{\mathbb{R}_{+}} \int_{\mathbb{R}^{N}} & {\left[(u-v) \Psi \partial_{t} \Phi+(g(t, x, u)-g(t, x, v)) \cdot(\nabla \Phi) \Psi\right.} \\
& +(g(t, x, u)-g(t, x, v)-f(s, y, u)+f(s, y, v)) \cdot(\nabla \Psi) \Phi \\
& +(F(s, y, u)-G(t, x, v)+\operatorname{div} g(t, x, u)-\operatorname{div} f(s, y, v)) \varphi] \\
& \times \operatorname{sign}(u-v) \mathrm{d} x \mathrm{~d} t \mathrm{~d} y \mathrm{~d} s \quad \geq 0 .
\end{aligned}
$$

Introduce a family of functions $\left\{Y_{\vartheta}\right\}_{\vartheta>0}$ as in (4.16). Let $M=\left\|\partial_{u} g\right\|_{\mathbf{L}^{\infty}\left(\Omega ; \mathbb{R}^{N}\right)}$ and define $\chi, \psi$ as in (4.17), for $\varepsilon, \theta, T_{o}, R>0, x_{o} \in \mathbb{R}^{N}$, (see also Figure 1). Remind that with these choices, equalities (4.18) still hold. Note that here the definition of the test function $\varphi$ is essentially the same as in the preceding proof; the only change is the definition of the constant $M$, that is now defined with reference to $g$. We also introduce as above the function $B(t, x, u, v)=M|u-v|+\operatorname{sign}(u-v)(g(t, x, u)-g(t, x, v)) \cdot \frac{x-x_{o}}{\left\|x-x_{o}\right\|}$ that is positive for all $(t, x, u, v) \in \Omega \times \mathbb{R}^{N}$, and we have:

$$
\begin{aligned}
& \int_{\mathbb{R}_{+}} \int_{\mathbb{R}^{N}} \int_{\mathbb{R}_{+}} \int_{\mathbb{R}^{N}}\left[(u-v) \partial_{t} \Phi+(g(t, x, u)-g(t, x, v)) \cdot \nabla \Phi\right] \Psi \operatorname{sign}(u-v) \mathrm{d} x \mathrm{~d} t \mathrm{~d} y \mathrm{~d} s \\
\leq & \int_{\mathbb{R}_{+}} \int_{\mathbb{R}^{N}} \int_{\mathbb{R}_{+}} \int_{\mathbb{R}^{N}}\left[|u-v| \chi^{\prime} \psi-B(t, x, u, v) \chi Y_{\theta}^{\prime}\right] \Psi \mathrm{d} x \mathrm{~d} t \mathrm{~d} y \mathrm{~d} s \\
\leq & \int_{\mathbb{R}_{+}} \int_{\mathbb{R}^{N}} \int_{\mathbb{R}_{+}} \int_{\mathbb{R}^{N}}|u-v| \chi^{\prime} \psi \Psi \mathrm{d} x \mathrm{~d} t \mathrm{~d} y \mathrm{~d} s .
\end{aligned}
$$

Thanks to the above estimate and (5.3), it results

$$
\begin{aligned}
\int_{\mathbb{R}_{+}} \int_{\mathbb{R}^{N}} \int_{\mathbb{R}_{+}} \int_{\mathbb{R}^{N}} & {\left[(u-v) \chi^{\prime} \psi \Psi\right.} \\
& +(g(t, x, u)-g(t, x, v)-f(s, y, u)+f(s, y, v)) \cdot(\nabla \Psi) \Phi \\
& +(F(s, y, u)-G(t, x, v)+\operatorname{div} g(t, x, u)-\operatorname{div} f(s, y, v)) \varphi] \geq 0, \\
& \times \operatorname{sign}(u-v) \mathrm{d} x \mathrm{~d} t \mathrm{~d} y \mathrm{~d} s \quad
\end{aligned}
$$


i.e. $I+J_{x}+J_{t}+K+L_{x}+L_{t} \geq 0$, where

$$
\begin{aligned}
& I=\int_{\mathbb{R}_{+}} \int_{\mathbb{R}^{N}} \int_{\mathbb{R}_{+}} \int_{\mathbb{R}^{N}}|u-v| \chi^{\prime} \psi \Psi \mathrm{d} x \mathrm{~d} t \mathrm{~d} y \mathrm{~d} s, \\
& J_{x}=\int_{\mathbb{R}_{+}} \int_{\mathbb{R}^{N}} \int_{\mathbb{R}_{+}} \int_{\mathbb{R}^{N}}(f(t, y, v)-f(t, y, u)+f(t, x, u)-f(t, x, v)) \cdot(\nabla \Psi) \Phi \\
& \times \operatorname{sign}(u-v) \mathrm{d} x \mathrm{~d} t \mathrm{~d} y \mathrm{~d} s, \\
& J_{t}=\int_{\mathbb{R}_{+}} \int_{\mathbb{R}^{N}} \int_{\mathbb{R}_{+}} \int_{\mathbb{R}^{N}}(f(s, y, v)-f(s, y, u)+f(t, y, u)-f(t, y, v)) \cdot(\nabla \Psi) \Phi \\
& \times \operatorname{sign}(u-v) \mathrm{d} x \mathrm{~d} t \mathrm{~d} y \mathrm{~d} s, \\
& K=\int_{\mathbb{R}_{+}} \int_{\mathbb{R}^{N}} \int_{\mathbb{R}_{+}} \int_{\mathbb{R}^{N}}((g-f)(t, x, u)-(g-f)(t, x, v)) \cdot(\nabla \Psi) \Phi \\
& \times \operatorname{sign}(u-v) \mathrm{d} x \mathrm{~d} t \mathrm{~d} y \mathrm{~d} s, \\
& L_{x}=\int_{\mathbb{R}_{+}} \int_{\mathbb{R}^{N}} \int_{\mathbb{R}_{+}} \int_{\mathbb{R}^{N}}(F(t, y, u)-G(t, x, v)+\operatorname{div} g(t, x, u)-\operatorname{div} f(t, y, v)) \varphi \\
& \times \operatorname{sign}(u-v) \mathrm{d} x \mathrm{~d} t \mathrm{~d} y \mathrm{~d} s, \\
& L_{t}=\int_{\mathbb{R}_{+}} \int_{\mathbb{R}^{N}} \int_{\mathbb{R}_{+}} \int_{\mathbb{R}^{N}}(F(s, y, u)-F(t, y, u)+\operatorname{div} f(t, y, v)-\operatorname{div} f(s, y, v)) \varphi \\
& \times \operatorname{sign}(u-v) \mathrm{d} x \mathrm{~d} t \mathrm{~d} y \mathrm{~d} s .
\end{aligned}
$$

Now, we choose $\Psi(t, x)=\nu(t) \mu(x)$ as in (4.19), (4.1), (4.2). Thanks to Lemma 5.2, Lemma 5.3 and Lemma 5.4 we obtain

$$
\begin{aligned}
\limsup _{\varepsilon, \eta, \lambda \rightarrow 0} I \leq & \int_{\left\|x-x_{o}\right\| \leq R+M T_{o}+\theta}|u(0, x)-v(0, x)| \mathrm{d} x \\
& -\int_{\left\|x-x_{o}\right\| \leq R+M\left(T_{o}-T\right)}|u(T, x)-v(T, x)| \mathrm{d} x \\
\limsup _{\varepsilon, \eta, \lambda \rightarrow 0} J_{x} \leq & N\left\|\nabla \partial_{u} f\right\|_{\mathbf{L}^{\infty}} \int_{0}^{T} \int_{B\left(x_{o}, R+M\left(T_{o}-t\right)+\theta\right)}|v(t, x)-u(t, x)| \mathrm{d} x \mathrm{~d} t \\
\limsup _{\varepsilon, \eta, \lambda \rightarrow 0} L_{x} \leq & \int_{0}^{T} \int_{B\left(x_{o}, R+M\left(T_{o}-t\right)+\theta\right)}\|((F-G)-\operatorname{div}(f-g))(t, y, \cdot)\|_{\mathbf{L}^{\infty}} \mathrm{d} y \mathrm{~d} t \\
& +\left(N\left\|\nabla \partial_{u} f\right\|_{\mathbf{L}^{\infty}}+\left\|\partial_{u} F\right\|_{\mathbf{L}^{\infty}}+\left\|\partial_{u}(F-G)\right\|_{\mathbf{L}^{\infty}}\right) \\
& \times \int_{0}^{T} \int_{B\left(x_{o}, R+M\left(T_{o}-t\right)+\theta\right)}|v(t, x)-u(t, x)| \mathrm{d} x \mathrm{~d} t
\end{aligned}
$$

Besides, we find that:

$$
\begin{aligned}
\left|J_{t}\right| & \leq \eta\left\|\partial_{t} \partial_{u} f\right\|_{\mathbf{L}^{\infty}} \int_{\mathbb{R}_{+}} \int_{\mathbb{R}^{N}} \int_{\mathbb{R}_{+}} \int_{\mathbb{R}^{N}}|u(t, x)-u(s, y)|\|\nabla \Psi\| \Phi \mathrm{d} x \mathrm{~d} t \mathrm{~d} y \mathrm{~d} s, \\
\left|L_{t}\right| & \leq \eta \omega_{N}\left(R+M T_{o}\right)^{N}(T+\varepsilon)\left(\left\|\partial_{t} \operatorname{div} f\right\|_{\mathbf{L}^{\infty}}+\left\|\partial_{t} F\right\|_{\mathbf{L}^{\infty}}\right),
\end{aligned}
$$

so that

$$
\limsup _{\eta \rightarrow 0}\left|J_{t}\right|=\limsup _{\eta \rightarrow 0}\left|L_{t}\right|=0 .
$$

In order to estimate $K$ as given in (5.6), we introduce a regularisation of the $y$ dependent functions. In fact, let $\rho_{\alpha}(z)=\frac{1}{\alpha} \rho\left(\frac{z}{\alpha}\right)$ and $\sigma_{\beta}(y)=\frac{1}{\beta^{N}} \sigma\left(\frac{y}{\beta}\right)$, where $\rho \in \mathbf{C}_{\mathbf{c}}^{\infty}\left(\mathbb{R} ; \overline{\mathbb{R}}_{+}\right)$and 
$\sigma \in \mathbf{C}_{\mathbf{c}}^{\infty}\left(\mathbb{R}^{N} ; \overline{\mathbb{R}}_{+}\right)$are such that $\|\rho\|_{\mathbf{L}^{1}(\mathbb{R} ; \mathbb{R})}=\|\sigma\|_{\mathbf{L}^{1}\left(\mathbb{R}^{N} ; \mathbb{R}\right)}=1$ and $\left.\operatorname{supp}(\rho) \subseteq\right]-1,1[$, $\operatorname{supp}(\sigma) \subseteq B(0,1)$. Then, introduce

$$
\begin{array}{rlrl}
P(w) & =(g-f)(t, x, w), & s_{\alpha}=\operatorname{sign} *_{u} \rho_{\alpha}, \\
\Upsilon_{\alpha}^{i}(w) & =s_{\alpha}(w-v)\left(P_{i}(w)-P_{i}(v)\right), & & u_{\beta}=\sigma_{\beta} *_{y} u, \\
\Upsilon^{i}(w) & =\operatorname{sign}(w-v)\left(P_{i}(w)-P_{i}(v)\right), & &
\end{array}
$$

so that we obtain

$$
\begin{aligned}
& \left\langle\Upsilon_{\alpha}^{i}\left(u_{\beta}\right)-\Upsilon_{\alpha}^{i}(u), \partial_{y_{i}} \varphi\right\rangle \\
= & \int_{\mathbb{R}^{N}} \int_{\mathbb{R}} \operatorname{sign}(w)\left(\rho_{\alpha}\left(u_{\beta}-v-w\right) P_{i}\left(u_{\beta}\right)-\rho_{\alpha}(u-v-w) P_{i}(u)\right) \partial_{y_{i}} \varphi \mathrm{d} w \mathrm{~d} y \\
& -\int_{\mathbb{R}^{N}} \int_{\mathbb{R}} \operatorname{sign}(w)\left(\rho_{\alpha}\left(u_{\beta}-v-w\right)-\rho_{\alpha}(u-v-w)\right) P_{i}(v) \partial_{y_{i}} \varphi \mathrm{d} w \mathrm{~d} y \\
= & \int_{\mathbb{R}^{N}} \int_{\mathbb{R}} \int_{u}^{u_{\beta}} \operatorname{sign}(w) \rho_{\alpha}^{\prime}(U-v-w)\left(P_{i}(U)-P_{i}(v)\right) \partial_{y_{i}} \varphi \mathrm{d} U \mathrm{~d} w \mathrm{~d} y \\
& +\int_{\mathbb{R}^{N}} \int_{\mathbb{R}} \int_{u}^{u_{\beta}} \operatorname{sign}(w) \rho_{\alpha}(U-v-w) P_{i}^{\prime}(U) \partial_{y_{i}} \varphi \mathrm{d} U \mathrm{~d} w \mathrm{~d} y .
\end{aligned}
$$

Now, we use the relation $\partial_{u} s_{\alpha}(u)=\frac{2}{\alpha} \rho\left(\frac{u}{\alpha}\right)$ to obtain

$$
\begin{aligned}
& \left|\left\langle\Upsilon_{\alpha}^{i}\left(u_{\beta}\right)-\Upsilon_{\alpha}^{i}(u), \partial_{y_{i}} \varphi\right\rangle\right| \\
\leq & \int_{\mathbb{R}^{N}} \frac{2}{\alpha} \sup _{U \in\left[\left(u, u_{\beta}\right)\right]}\left(\rho\left(\frac{U-v}{\alpha}\right)\left(P_{i}(U)-P_{i}(v)\right)\right) \min \left\{2 \alpha,\left|u-u_{\beta}\right|\right\} \partial_{y_{i}} \varphi \mathrm{d} y \\
& +\int_{\mathbb{R}^{N}} \int_{u}^{u_{\beta}}\left|P_{i}^{\prime}(U)\right| \partial_{y_{i}} \varphi \mathrm{d} U \mathrm{~d} y .
\end{aligned}
$$

When $\alpha$ tends to 0 , thanks to the Dominated Convergence Theorem, we obtain

$$
\left|\left\langle\Upsilon^{i}\left(u_{\beta}\right)-\Upsilon^{i}(u), \partial_{y_{i}} \varphi\right\rangle\right| \leq \int_{\mathbb{R}^{N}}\left|u-u_{\beta}\right|\left\|P_{i}^{\prime}\right\|_{\mathbf{L}^{\infty}} \partial_{y_{i}} \varphi \mathrm{d} y
$$

Applying the Dominated Convergence Theorem again, we see that

$$
\begin{aligned}
& \lim _{\beta \rightarrow 0} \lim _{\alpha \rightarrow 0}\left\langle\Upsilon_{\alpha}^{i}\left(u_{\beta}\right), \partial_{y_{i}} \varphi\right\rangle=\left\langle\Upsilon^{i}(u), \partial_{y_{i}} \varphi\right\rangle, \\
& \lim _{\beta \rightarrow 0} \lim _{\alpha \rightarrow 0}\left\langle\Upsilon_{\alpha}\left(u_{\beta}\right), \nabla_{y} \varphi\right\rangle=\left\langle\Upsilon(u), \nabla_{y} \varphi\right\rangle .
\end{aligned}
$$

Consequently, it is sufficient to find a bound independent of $\alpha$ and $\beta$ on $K_{\alpha, \beta}$, where

$$
K_{\alpha, \beta}=-\int_{\mathbb{R}_{+}} \int_{\mathbb{R}^{N}} \int_{\mathbb{R}_{+}} \int_{\mathbb{R}^{N}} \Upsilon_{\alpha}\left(u_{\beta}\right) \cdot \nabla_{y} \varphi \mathrm{d} x \mathrm{~d} t \mathrm{~d} y \mathrm{~d} s
$$

Integrating by parts, we get

$$
\begin{aligned}
K_{\alpha, \beta}= & \int_{\mathbb{R}_{+}} \int_{\mathbb{R}^{N}} \int_{\mathbb{R}_{+}} \int_{\mathbb{R}^{N}} \operatorname{Div}_{y} \Upsilon_{\alpha}\left(u_{\beta}\right) \varphi \mathrm{d} x \mathrm{~d} t \mathrm{~d} y \mathrm{~d} s \\
= & \int_{\mathbb{R}_{+}} \int_{\mathbb{R}^{N}} \int_{\mathbb{R}_{+}} \int_{\mathbb{R}^{N}} \partial_{u} s_{\alpha}\left(u_{\beta}-v\right) \nabla u_{\beta} \cdot\left((g-f)\left(t, x, u_{\beta}\right)-(g-f)(t, x, v)\right) \varphi \mathrm{d} x \mathrm{~d} t \mathrm{~d} y \mathrm{~d} s \\
& +\int_{\mathbb{R}_{+}} \int_{\mathbb{R}^{N}} \int_{\mathbb{R}_{+}} \int_{\mathbb{R}^{N}} s_{\alpha}\left(u_{\beta}-v\right)\left(\partial_{u}(g-f)\left(t, x, u_{\beta}\right) \cdot \nabla u_{\beta}\right) \varphi \mathrm{d} x \mathrm{~d} t \mathrm{~d} y \mathrm{~d} s \\
= & K_{1}+K_{2} .
\end{aligned}
$$

We now search a bound for each term of the sum above. 
- For $K_{1}$, recall that $\partial_{u} s_{\alpha}(u)=\frac{2}{\alpha} \rho\left(\frac{u}{\alpha}\right)$. Hence, by Dominated Convergence Theorem, we get that $K_{1} \rightarrow 0$ when $\alpha \rightarrow 0$. Indeed,

$$
\begin{aligned}
& \left|\frac{2}{\alpha} \rho\left(\frac{u_{\beta}-v}{\alpha}\right) \nabla u_{\beta} \cdot\left((g-f)\left(t, x, u_{\beta}\right)-(g-f)(t, x, v)\right) \varphi\right| \\
\leq & \frac{2}{\alpha} \rho\left(\frac{u_{\beta}-v}{\alpha}\right) \varphi\left\|\nabla u_{\beta}(s, y)\right\| \int_{v}^{u_{\beta}}\left\|\partial_{u}(f-g)(t, x, w)\right\| \mathrm{d} w \\
\leq & 2\|\rho\|_{\mathbf{L}^{\infty}(\mathbb{R} ; \mathbb{R})}\left\|\nabla u_{\beta}(s, y)\right\|\left\|\partial_{u}(f-g)\right\|_{\mathbf{L}^{\infty}\left(\Omega ; \mathbb{R}^{N}\right)} \varphi \quad \in \mathbf{L}^{\mathbf{1}}\left(\left(\mathbb{R}_{+} \times \mathbb{R}^{N}\right)^{2} ; \mathbb{R}\right) .
\end{aligned}
$$

- Concerning $K_{2}$,

$$
\begin{aligned}
K_{2} & \leq\left\|\partial_{u}(f-g)\right\|_{\mathbf{L}^{\infty}\left(\Omega ; \mathbb{R}^{N}\right)} \int_{0}^{T+\varepsilon+\eta} \int_{\mathbb{R}^{N}}\left\|\nabla u_{\beta}(s, y)\right\| \mathrm{d} y \mathrm{~d} s \\
& \leq\left\|\partial_{u}(f-g)\right\|_{\mathbf{L}^{\infty}\left(\Omega ; \mathbb{R}^{N}\right)} \int_{0}^{T+\varepsilon+\eta} \operatorname{TV}\left(u_{\beta}(t)\right) \mathrm{d} t .
\end{aligned}
$$

Finally, letting $\alpha, \beta \rightarrow 0$ and $\varepsilon, \eta, \lambda \rightarrow 0$, thanks to [1], Proposition 3.7], we get

$$
\limsup _{\varepsilon, \eta, \lambda \rightarrow 0} K \leq\left\|\partial_{u}(f-g)\right\|_{\mathbf{L}^{\infty}} \int_{0}^{T} \operatorname{TV}(u(t)) \mathrm{d} t .
$$

Now, we collate the estimates obtained in (5.8), (5.9), (5.10), (5.11) and (5.12). Remark the order in which we pass to the various limits: first $\varepsilon, \eta, \theta \rightarrow 0$ and, after, $\lambda \rightarrow 0$. Therefore, we get

$$
\begin{aligned}
& \int_{B\left(x_{o}, R+M\left(T_{o}-T\right)\right)}|u(T, x)-v(T, x)| \mathrm{d} x \\
& \leq \int_{B\left(x_{o}, R+M T_{o}\right)}|u(0, x)-v(0, x)| \mathrm{d} x \\
& \quad+\left[2 N\left\|\nabla \partial_{u} f\right\|_{\mathbf{L}^{\infty}}+\left\|\partial_{u} F\right\|_{\mathbf{L}^{\infty}}+\left\|\partial_{u}(F-G)\right\|_{\mathbf{L}^{\infty}}\right] \\
& \quad \times \int_{0}^{T} \int_{B\left(x_{o}, R+M\left(T_{o}-t\right)\right)}|v(t, x)-u(t, x)| \mathrm{d} x \mathrm{~d} t \\
& +\left[\left\|\partial_{u}(f-g)\right\|_{\mathbf{L}^{\infty}} \int_{0}^{T} \operatorname{TV}(u(t)) \mathrm{d} t\right. \\
& \left.\quad+\int_{0}^{T} \int_{B\left(x_{o}, R+M\left(T_{o}-t\right)\right)}\|((F-G)-\operatorname{div}(f-g))(t, y, \cdot)\|_{\mathbf{L}^{\infty}} \mathrm{d} y \mathrm{~d} t\right]
\end{aligned}
$$

or equivalently

$$
A^{\prime}(T) \leq A^{\prime}(0)+\kappa A(T)+S(T)
$$

where

$$
\begin{aligned}
A(T)= & \int_{0}^{T} \int_{B\left(x_{o}, R+M\left(T_{o}-t\right)\right)}|v(t, x)-u(t, x)| \mathrm{d} x \mathrm{~d} t \\
\kappa= & 2 N\left\|\nabla \partial_{u} f\right\|_{\mathbf{L}^{\infty}}+\left\|\partial_{u} F\right\|_{\mathbf{L}^{\infty}}+\left\|\partial_{u}(F-G)\right\|_{\mathbf{L}^{\infty}} \\
S(T)= & \left\|\partial_{u}(f-g)\right\|_{\mathbf{L}^{\infty}} \int_{0}^{T} \operatorname{TV}(u(t)) \mathrm{d} t \\
& \quad+\int_{0}^{T} \int_{B\left(x_{o}, R+M\left(T_{o}-t\right)\right)}\|((F-G)-\operatorname{div}(f-g))(t, y, \cdot)\|_{\mathbf{L}^{\infty}} \mathrm{d} y \mathrm{~d} t .
\end{aligned}
$$


The bound 2.5) on TV $(u(t))$ gives:

$$
S(T) \leq \frac{e^{\kappa_{o} T}-1}{\kappa_{o}} a+\int_{0}^{T} \frac{e^{\kappa_{o}(T-t)}-1}{\kappa_{o}} b(t) \mathrm{d} t+\int_{0}^{T} c(t) \mathrm{d} t
$$

where $\kappa_{o}$ is defined in (2.4) and

$$
\begin{aligned}
a & =\left\|\partial_{u}(f-g)\right\|_{\mathbf{L}^{\infty}} \operatorname{TV}\left(u_{o}\right), \\
b(t) & =N W_{N}\left\|\partial_{u}(f-g)\right\|_{\mathbf{L}^{\infty}} \int_{\mathbb{R}^{N}}\|\nabla(F-\operatorname{div} f)(t, x, \cdot)\|_{\mathbf{L}^{\infty}} \mathrm{d} x, \\
c(t) & =\int_{B\left(x_{o}, R+M\left(T_{o}-t\right)\right)}\|((F-G)-\operatorname{div}(f-g))(t, y, \cdot)\|_{\mathbf{L}^{\infty}} \mathrm{d} y,
\end{aligned}
$$

since $T \leq T_{o}$. Consequently

$$
A^{\prime}(T) \leq A^{\prime}(0)+\kappa A(T)+\left(\frac{e^{\kappa_{o} T}-1}{\kappa_{o}} a+\int_{0}^{T} \frac{e^{\kappa_{o}(T-t)}-1}{\kappa_{o}} b(t) \mathrm{d} t+\int_{0}^{T} c(t) \mathrm{d} t\right) .
$$

By a Gronwall type argument, if $\kappa_{o}=\kappa$, we get

$$
A^{\prime}(T) \leq e^{\kappa T} A^{\prime}(0)+T e^{\kappa T} a+\left(\int_{0}^{T}(T-t) e^{\kappa(T-t)} b(t) \mathrm{d} t\right)\left(\int_{0}^{T} e^{\kappa(T-t)} c(t) \mathrm{d} t\right)
$$

yielding

$$
\begin{aligned}
& \int_{\left\|x-x_{o}\right\| \leq R}|u(T, x)-v(T, x)| \mathrm{d} x \leq e^{\kappa T} \int_{\left\|x-x_{o}\right\| \leq R+M T}\left|u_{o}(x)-v_{o}(x)\right| \mathrm{d} x \\
+ & T e^{\kappa T} \operatorname{TV}\left(u_{o}\right)\left\|\partial_{u}(f-g)\right\|_{\mathbf{L}^{\infty}} \\
+ & N W_{N}\left(\int_{0}^{T}(T-t) e^{\kappa(T-t)} \int_{\mathbb{R}^{N}}\|\nabla(F-\operatorname{div} f)(t, x, \cdot)\|_{\mathbf{L}^{\infty}} \mathrm{d} x \mathrm{~d} t\right)\left\|\partial_{u}(f-g)\right\|_{\mathbf{L}^{\infty}} \\
+ & \int_{0}^{T} e^{\kappa(T-t)} \int_{\left\|x-x_{o}\right\| \leq R+M(T-t)}\|((F-G)-\operatorname{div}(f-g))(t, x, \cdot)\|_{\mathbf{L}^{\infty}} \mathrm{d} x \mathrm{~d} t
\end{aligned}
$$

while, in the case $\kappa_{o} \neq \kappa$, we have

$$
A^{\prime}(T) \leq e^{\kappa T} A^{\prime}(0)+\frac{e^{\kappa_{o} T}-e^{\kappa T}}{\kappa_{o}-\kappa} a+\int_{0}^{T} \frac{e^{\kappa_{o}(T-t)}-e^{\kappa(T-t)}}{\kappa_{o}-\kappa} b(t) \mathrm{d} t+\int_{0}^{T} e^{\kappa(T-t)} c(t) \mathrm{d} t .
$$

Taking $T=T_{o}$, we finally obtain the result.

Remark 5.1 Assuming that also $(g, G)$ satisfies (H2), allows us to exchange the role of $u$ and $v$ in (5.14). Let

$$
\begin{aligned}
\tilde{\kappa}_{o} & =N W_{N}\left((2 N+1)\left\|\nabla \partial_{u} g\right\|_{\mathbf{L}^{\infty}}+\left\|\partial_{u} G\right\|_{\mathbf{L}^{\infty}}\right) \\
\tilde{a} & =\left\|\partial_{u}(f-g)\right\|_{\mathbf{L}^{\infty}} \operatorname{TV}\left(v_{o}\right) \\
\tilde{b}(t) & =\left\|\partial_{u}(f-g)\right\|_{\mathbf{L}^{\infty}} N W_{N} \int_{\mathbb{R}^{N}}\|\nabla(G-\operatorname{div} g)(t, x, \cdot)\|_{\mathbf{L}^{\infty}} \mathrm{d} x \\
\tilde{\kappa} & =2 N\left\|\nabla \partial_{u} g\right\|_{\mathbf{L}^{\infty}}+\left\|\partial_{u} G\right\|_{\mathbf{L}^{\infty}}+\left\|\partial_{u}(F-G)\right\|_{\mathbf{L}^{\infty}},
\end{aligned}
$$


and repeating the same computations as above, we obtain

$$
A^{\prime}(T) \leq A(0)+\tilde{\kappa} A(T)+\left(\frac{e^{\tilde{\kappa}_{o} T}-1}{\tilde{\kappa}_{o}} \tilde{a}+\int_{0}^{T} \frac{e^{\tilde{\kappa}_{o}(T-t)}-1}{\tilde{\kappa}_{o}} \tilde{b}(t) \mathrm{d} t+\int_{0}^{T} c(t) \mathrm{d} t\right)
$$

so that, finally,

$$
\begin{aligned}
A^{\prime}(T) \leq & A^{\prime}(0)+\min (\kappa, \tilde{\kappa}) A(T)+\max \left[\frac{e^{\kappa_{o} T}-1}{\kappa_{o}} a+\int_{0}^{T} \frac{e^{\kappa_{o}(T-t)}-1}{\kappa_{o}} b(t) \mathrm{d} t,\right. \\
& \left.\frac{e^{\tilde{\kappa}_{o} T}-1}{\tilde{\kappa}_{O}} \tilde{a}+\int_{0}^{T} \frac{e^{\tilde{\kappa}_{o}(T-t)}-1}{\tilde{\kappa}_{O}} \tilde{b}(t) \mathrm{d} t\right]+\int_{0}^{T} c(t) \mathrm{d} t .
\end{aligned}
$$

We collect below some lemmas that were used in the previous proof. The first one reminds a part of the proof of [3, Theorem 2.1].

Lemma 5.2 Let I be defined as in (5.4). Then,

$$
\begin{aligned}
\limsup _{\varepsilon \rightarrow 0} I \leq & \int_{\left\|x-x_{o}\right\| \leq R+M T_{o}+\theta}|u(0, x)-v(0, x)| \mathrm{d} x \\
& -\int_{\left\|x-x_{o}\right\| \leq R+M\left(T_{o}-T\right)}|u(T, x)-v(T, x)| \mathrm{d} x+2 \sup _{\tau \in\{0, T\}} \operatorname{TV}(u(\tau)) \lambda \\
& +2 \sup _{\substack{t \in\{0, T\} \\
s \in] t, t+\eta[}} \int_{\left\|y-x_{o}\right\| \leq R+\lambda+M\left(T_{o}-t\right)+\theta}|u(t, y)-u(s, y)| \mathrm{d} y .
\end{aligned}
$$

Proof. By the triangle inequality $I \leq I_{1}+I_{2}+I_{3}$, with

$$
\begin{aligned}
I_{1} & =\int_{\mathbb{R}_{+}} \int_{\mathbb{R}^{N}} \int_{\mathbb{R}_{+}} \int_{\mathbb{R}^{N}}|u(t, x)-v(t, x)| \chi^{\prime}(t) \psi(t, x) \Psi(t-s, x-y) \mathrm{d} x \mathrm{~d} t \mathrm{~d} y \mathrm{~d} s, \\
I_{2} & =\int_{\mathbb{R}_{+}} \int_{\mathbb{R}^{N}} \int_{\mathbb{R}_{+}} \int_{\mathbb{R}^{N}}|u(t, x)-u(t, y)|\left|\chi^{\prime}(t)\right| \psi(t, x) \Psi(t-s, x-y) \mathrm{d} x \mathrm{~d} t \mathrm{~d} y \mathrm{~d} s, \\
I_{3} & =\int_{\mathbb{R}_{+}} \int_{\mathbb{R}^{N}} \int_{\mathbb{R}_{+}} \int_{\mathbb{R}^{N}}|u(t, y)-u(s, y)|\left|\chi^{\prime}(t)\right| \psi(t, x) \Psi(t-s, x-y) \mathrm{d} x \mathrm{~d} t \mathrm{~d} y \mathrm{~d} s .
\end{aligned}
$$

Then,

$$
\begin{aligned}
I_{1}= & \int_{\mathbb{R}_{+}} \int_{\mathbb{R}^{N}}|u(t, x)-v(t, x)|\left(Y_{\varepsilon}^{\prime}(t)-Y_{\varepsilon}^{\prime}(t-T)\right) \psi(t, x) \mathrm{d} x \mathrm{~d} t \\
\leq & \int_{\mathbb{R}_{+}} \int_{\left\|x-x_{o}\right\| \leq R+M\left(T_{o}-t\right)+\theta}|u(t, x)-v(t, x)| Y_{\varepsilon}^{\prime}(t) \mathrm{d} x \mathrm{~d} t \\
& -\int_{\mathbb{R}_{+}} \int_{\left\|x-x_{o}\right\| \leq R+M\left(T_{o}-t\right)}|u(t, x)-v(t, x)| Y_{\varepsilon}^{\prime}(t-T) \mathrm{d} x \mathrm{~d} t
\end{aligned}
$$

and by the $\mathbf{L}^{\mathbf{1}}$ right continuity of $u$ and $v$ in time, thanks to Theorem 2.3

$$
\begin{aligned}
\limsup _{\varepsilon \rightarrow 0} I_{1} \leq & \int_{\left\|x-x_{o}\right\| \leq R+M T_{o}+\theta}|u(0, x)-v(0, x)| \mathrm{d} x \\
& -\int_{\left\|x-x_{o}\right\| \leq R+M\left(T_{o}-T\right)}|u(T, x)-v(T, x)| \mathrm{d} x .
\end{aligned}
$$


For $I_{2}$ and $I_{3}$, we have

$$
\begin{aligned}
& I_{2} \leq \int_{\mathbb{R}_{+}} \int_{\mathbb{R}^{N}} \int_{\left\|x-x_{o}\right\| \leq R+M\left(T_{o}-t\right)+\theta}|u(t, x)-u(t, y)|\left(Y_{\varepsilon}^{\prime}(t)+Y_{\varepsilon}^{\prime}(t-T)\right) \mu \mathrm{d} x \mathrm{~d} y \mathrm{~d} t, \\
& I_{3} \leq \int_{\mathbb{R}_{+}} \int_{\mathbb{R}_{+}} \int_{\left\|y-x_{o}\right\| \leq R+\lambda+M\left(T_{o}-t\right)+\theta}|u(t, y)-u(s, y)|\left(Y_{\varepsilon}^{\prime}(t)+Y_{\varepsilon}^{\prime}(t-T)\right) \nu \mathrm{d} y \mathrm{~d} s \mathrm{~d} t .
\end{aligned}
$$

As $\varepsilon \rightarrow 0$, we use on the one hand the $\mathbf{L}^{\mathbf{1}}$ right continuity in time of $u$, thanks to Theorem 2.3, and on the other hand that $u(t) \in \mathbf{B V}\left(\mathbb{R}^{N} ; \mathbb{R}\right)$, thanks to Theorem 2.5. In particular, we can use (4.11) to obtain

$$
\begin{aligned}
\limsup _{\varepsilon \rightarrow 0} I_{2} & \leq \sum_{t=0, T} \sup _{\|h\| \leq \lambda} \int_{\left\|x-x_{o}\right\| \leq R+M\left(T_{o}-t\right)+\theta}|u(t, x)-u(t, x+h)| \mathrm{d} x \\
& \leq 2 \sup _{\substack{\|h\| \leq \lambda \\
t \in\{0, T\}}} \int_{\left\|x-x_{o}\right\| \leq R+M\left(T_{o}-t\right)+\theta}|u(t, x)-u(t, x+h)| \mathrm{d} x \\
& \leq 2 \sup _{t \in\{0, T\}} \operatorname{TV}(u(t)) \lambda, \\
\limsup _{\varepsilon \rightarrow 0} I_{3} & \leq \sum_{t=0, T} \sup _{s \in t t, t+\eta[} \int_{\left\|y-x_{o}\right\| \leq R+\lambda+M\left(T_{o}-t\right)+\theta}|u(t, y)-u(s, y)| \mathrm{d} y \\
& \leq 2 \sup _{\substack{t \in\{0, T\} \\
s \in] t, t+\eta[}} \int_{\left\|y-x_{o}\right\| \leq R+\lambda+M\left(T_{o}-t\right)+\theta}|u(t, y)-u(s, y)| \mathrm{d} y .
\end{aligned}
$$

Lemma 5.3 Let $J_{x}$ be defined as in (5.5). Then,

$$
\begin{aligned}
\limsup _{\varepsilon \rightarrow 0} J_{x} \leq & N\left\|\nabla \partial_{u} f\right\|_{\mathbf{L}^{\infty}} \int_{0}^{T} \int_{B\left(x_{o}, R+M\left(T_{o}-t\right)+\theta\right)}|v(t, x)-u(t, x)| \mathrm{d} x \mathrm{~d} t \\
& +N T\left\|\nabla \partial_{u} f\right\|_{\mathbf{L}^{\infty}} \sup _{\tau \in[0, T]} \operatorname{TV}(u(\tau)) \lambda \\
& +N T\left\|\nabla \partial_{u} f\right\|_{\mathbf{L}^{\infty}} \sup _{\substack{t \in[0, T] \\
s \in t, t+\eta[}} \int_{\left\|y-x_{o}\right\| \leq R+\lambda+M\left(T_{o}-t\right)+\theta}|u(t, y)-u(s, y)| \mathrm{d} y .
\end{aligned}
$$

Proof. By assumptions (H1), $f \in \mathbf{C}^{2}\left(\Omega ; \mathbb{R}^{N}\right)$ and therefore

$$
\begin{aligned}
& \|f(t, y, v)-f(t, y, u)+f(t, x, u)-f(t, x, v)\| \\
= & \left\|\int_{u(s, y)}^{v(t, x)} \int_{0}^{1} \nabla \partial_{u} f(t, x(1-r)+r y, w) \cdot(y-x) \mathrm{d} r \mathrm{~d} w\right\| \\
\leq & \left\|\nabla \partial_{u} f\right\|_{\mathbf{L}^{\infty}}\|x-y\||v(t, x)-u(s, y)| .
\end{aligned}
$$

Then,

$$
J_{x} \leq\left\|\nabla \partial_{u} f\right\|_{\mathbf{L}^{\infty}} \int_{\mathbb{R}_{+}} \int_{\mathbb{R}^{N}} \int_{\mathbb{R}_{+}} \int_{\mathbb{R}^{N}}|v(t, x)-u(s, y)|\|x-y\|\|\nabla \mu\| \nu \chi \psi \mathrm{d} x \mathrm{~d} t \mathrm{~d} y \mathrm{~d} s .
$$


Similarly to the proof of Lemma 5.2, we apply the triangle inequality and obtain $J_{x} \leq$ $J_{1}+J_{2}+J_{3}$ where

$$
\begin{aligned}
J_{1} & =\left\|\nabla \partial_{u} f\right\|_{\mathbf{L}^{\infty}} \int_{\mathbb{R}_{+}} \int_{\mathbb{R}^{N}} \int_{\mathbb{R}_{+}} \int_{\mathbb{R}^{N}}|v(t, x)-u(t, x)|\|x-y\|\|\nabla \mu\| \nu \chi \psi \mathrm{d} x \mathrm{~d} t \mathrm{~d} y \mathrm{~d} s, \\
J_{2} & =\left\|\nabla \partial_{u} f\right\|_{\mathbf{L}^{\infty}} \int_{\mathbb{R}_{+}} \int_{\mathbb{R}^{N}} \int_{\mathbb{R}_{+}} \int_{\mathbb{R}^{N}}|u(t, x)-u(t, y)|\|x-y\|\|\nabla \mu\| \nu \chi \psi \mathrm{d} x \mathrm{~d} t \mathrm{~d} y \mathrm{~d} s, \\
J_{3} & =\left\|\nabla \partial_{u} f\right\|_{\mathbf{L}^{\infty}} \int_{\mathbb{R}_{+}} \int_{\mathbb{R}^{N}} \int_{\mathbb{R}_{+}} \int_{\mathbb{R}^{N}}|u(t, y)-u(s, y)|\|x-y\|\|\nabla \mu\| \nu \chi \psi \mathrm{d} x \mathrm{~d} t \mathrm{~d} y \mathrm{~d} s .
\end{aligned}
$$

For $J_{1}$, we have, thanks to (4.5)

$$
J_{1} \leq N\left\|\nabla \partial_{u} f\right\|_{\mathbf{L}^{\infty}} \int_{0}^{T+\varepsilon} \int_{B\left(x_{o}, R+M\left(T_{o}-t\right)+\theta\right)}|v(t, x)-u(t, x)| \mathrm{d} x \mathrm{~d} t .
$$

For $J_{2}$, we have

$$
\begin{aligned}
J_{2} & \leq N\left\|\nabla \partial_{u} f\right\|_{\mathbf{L}^{\infty}} \int_{0}^{T+\varepsilon} \sup _{\|h\| \leq \lambda} \int_{\left\|x-x_{o}\right\| \leq R+M\left(T_{o}-t\right)+\theta}|u(t, x)-u(t, x+h)| \mathrm{d} x \mathrm{~d} t \\
& \leq N\left\|\nabla \partial_{u} f\right\|_{\mathbf{L}^{\infty}}(T+\varepsilon) \sup _{\tau \in[0, T+\varepsilon]} \operatorname{TV}(u(\tau)) \lambda,
\end{aligned}
$$

and for $J_{3}$

$$
\begin{aligned}
J_{3} & \leq N\left\|\nabla \partial_{u} f\right\|_{\mathbf{L}^{\infty}} \int_{0}^{T+\varepsilon} \sup _{s \in] t, t+\eta[} \int_{\left\|x-x_{o}\right\| \leq R+\lambda+M\left(T_{o}-t\right)+\theta}|u(t, y)-u(s, y)| \mathrm{d} y \mathrm{~d} t \\
& \leq N\left\|\nabla \partial_{u} f\right\|_{\mathbf{L}^{\infty}}(T+\varepsilon) \sup _{\substack{t \in[0, T+\varepsilon] \\
s \in] t, t+\eta]}} \int_{\left\|y-x_{o}\right\| \leq R+\lambda+M\left(T_{o}-t\right)+\theta}|u(t, y)-u(s, y)| \mathrm{d} y .
\end{aligned}
$$

In particular, letting $\lambda, \eta, \varepsilon, \theta \rightarrow 0$, we prove that $J_{2}, J_{3} \rightarrow 0$ and

$$
\limsup _{\lambda, \eta, \varepsilon, \theta \rightarrow 0} J_{1} \leq N\left\|\nabla \partial_{u} f\right\|_{\mathbf{L}^{\infty}} \int_{0}^{T} \int_{B\left(x_{o}, R+M\left(T_{o}-t\right)\right)}|v(t, x)-u(t, x)| \mathrm{d} x \mathrm{~d} t
$$

completing the proof.

Lemma 5.4 Let $L_{x}$ be defined as in (5.7) and $M_{1}$ as in (4.20). Then

$$
\begin{aligned}
\limsup _{\varepsilon \rightarrow 0} L_{x} \leq & T \int_{0}^{T} \int_{\left\|x-x_{o}\right\| \leq R+M\left(T_{o}-t\right)+\theta}\|((F-G)-\operatorname{div}(f-g))(t, x, \cdot)\|_{\mathbf{L}^{\infty}} \mathrm{d} x \mathrm{~d} t \\
+ & \left(N\left\|\nabla \partial_{u} f\right\|_{\mathbf{L}^{\infty}}+\left\|\partial_{u} F\right\|_{\mathbf{L}^{\infty}}+\left\|\partial_{u}(F-G)\right\|_{\mathbf{L}^{\infty}}\right) \\
\times & {\left[\int_{0}^{T} \int_{B\left(x_{o}, R+M\left(T_{o}-t\right)+\theta\right)}|v(t, x)-u(t, x)| \mathrm{d} x \mathrm{~d} t\right.} \\
& +T \sup _{\tau \in[0, T]} \operatorname{TV}(u(\tau)) \lambda \\
& \left.+T \sup _{t \in[0, T]} \int_{\left\|y-x_{o}\right\| \leq R+\lambda+M\left(T_{o}-t\right)+\theta}|u(t, y)-u(s, y)| \mathrm{d} y\right] \\
+\lambda & M_{1} \int_{0}^{T} \int_{\mathbb{R}^{N}}\|\nabla(F-\operatorname{div} f)(t, x, \cdot)\|_{\mathbf{L}^{\infty}} \mathrm{d} x \mathrm{~d} t .
\end{aligned}
$$


Proof. Let

$$
\begin{aligned}
L_{1} & =\int_{\mathbb{R}_{+}} \int_{\mathbb{R}^{N}} \int_{\mathbb{R}_{+}} \int_{\mathbb{R}^{N}}((F-G)-\operatorname{div}(f-g))(t, x, u) \varphi \operatorname{sign}(u-v) \mathrm{d} x \mathrm{~d} t \mathrm{~d} y \mathrm{~d} s, \\
L_{2} & =\int_{\mathbb{R}_{+}} \int_{\mathbb{R}^{N}} \int_{\mathbb{R}_{+}} \int_{\mathbb{R}^{N}}((F-G)(t, x, v)-(F-G)(t, x, u)) \varphi \operatorname{sign}(u-v) \mathrm{d} x \mathrm{~d} t \mathrm{~d} y \mathrm{~d} s, \\
L_{3} & =\int_{\mathbb{R}_{+}} \int_{\mathbb{R}^{N}} \int_{\mathbb{R}_{+}} \int_{\mathbb{R}^{N}}(F(t, y, u)-F(t, y, v)+\operatorname{div} f(t, x, u)-\operatorname{div} f(t, x, v)) \varphi \\
L_{4} & =\int_{\mathbb{R}_{+}} \int_{\mathbb{R}^{N}} \int_{\mathbb{R}_{+}} \int_{\mathbb{R}^{N}}((F-\operatorname{div} f)(t, y, v)-(F-\operatorname{div} f)(t, x, v)) \varphi \operatorname{sign}(u-v) \mathrm{d} x \mathrm{~d} t \mathrm{~d} y \mathrm{~d} s,
\end{aligned}
$$

so that $L_{x}=L_{1}+L_{2}+L_{3}+L_{4}$. Clearly,

$$
L_{1} \leq \int_{0}^{T+\varepsilon} \int_{\left\|x-x_{o}\right\| \leq R+M\left(T_{o}-t\right)+\theta}\|((G-F)-\operatorname{div}(f-g))(t, x, \cdot)\|_{\mathbf{L}^{\infty}} \mathrm{d} x \mathrm{~d} t .
$$

For $L_{2}$ and $L_{3}$, we have

$$
\begin{aligned}
L_{2} & \leq\left\|\partial_{u}(F-G)\right\|_{\mathbf{L}^{\infty}} \int_{\mathbb{R}_{+}} \int_{\mathbb{R}^{N}} \int_{\mathbb{R}_{+}} \int_{\mathbb{R}^{N}}|u(s, y)-v(t, x)| \varphi \mathrm{d} x \mathrm{~d} t \mathrm{~d} y \mathrm{~d} s, \\
L_{3} & =\int_{\mathbb{R}_{+}} \int_{\mathbb{R}^{N}} \int_{\mathbb{R}_{+}} \int_{\mathbb{R}^{N}} \operatorname{sign}(u-v)\left(\int_{v}^{u}\left(\partial_{u} \operatorname{div} f(t, x, w)+\partial_{u} F(t, y, w)\right) \mathrm{d} w\right) \varphi \mathrm{d} x \mathrm{~d} t \mathrm{~d} y \mathrm{~d} s \\
& \leq\left(N\left\|\nabla \partial_{u} f\right\|_{\mathbf{L}^{\infty}}+\left\|\partial_{u} F\right\|_{\mathbf{L}^{\infty}}\right) \int_{\mathbb{R}_{+}} \int_{\mathbb{R}^{N}} \int_{\mathbb{R}_{+}} \int_{\mathbb{R}^{N}}|v(t, x)-u(s, y)| \varphi \mathrm{d} x \mathrm{~d} t \mathrm{~d} y \mathrm{~d} s .
\end{aligned}
$$

Proceeding as for $J_{x}$, we find the following bound for $\iiint \int|v(t, x)-u(s, y)| \varphi$ in $L_{2}, L_{3}$.

$$
\begin{aligned}
L_{2}+L_{3} \leq & \left(N\left\|\nabla \partial_{u} f\right\|_{\mathbf{L}^{\infty}}+\left\|\partial_{u} F\right\|_{\mathbf{L}^{\infty}}+\left\|\partial_{u}(F-G)\right\|_{\mathbf{L}^{\infty}}\right) \\
& \times\left[\int_{0}^{T+\varepsilon} \int_{B\left(x_{o}, R+M\left(T_{o}-t\right)+\theta\right)}|v(t, x)-u(t, x)| \mathrm{d} x \mathrm{~d} t+(T+\varepsilon) \sup _{\tau \in[0, T+\varepsilon]} \operatorname{TV}(u(\tau)) \lambda\right. \\
& \left.\quad+(T+\varepsilon) \sup _{\substack{t \in[0, T+\varepsilon] \\
s \in] t, t+\eta]}} \int_{\left\|y-x_{o}\right\| \leq R+\lambda+M\left(T_{o}-t\right)+\theta}|u(t, y)-u(s, y)| \mathrm{d} y\right] .
\end{aligned}
$$

For $L_{4}$ we have

$$
\begin{aligned}
L_{4}= & \int_{\mathbb{R}_{+}} \int_{\mathbb{R}^{N}} \int_{\mathbb{R}_{+}} \int_{\mathbb{R}^{N}}\left[\int_{0}^{1} \nabla(F-\operatorname{div} f)(t, r x+(1-r) y, v) \cdot(y-x) \mathrm{d} r\right] \varphi \\
& \times \operatorname{sign}(u-v) \mathrm{d} x \mathrm{~d} t \mathrm{~d} y \mathrm{~d} s \\
\leq & \lambda M_{1} \int_{0}^{T+\varepsilon} \int_{\mathbb{R}^{N}}\|\nabla(F-\operatorname{div} f)(t, x, \cdot)\|_{\mathbf{L}^{\infty}} \mathrm{d} x \mathrm{~d} t .
\end{aligned}
$$

To complete the proof, it is sufficient to note that $L_{x}=L_{1}+L_{2}+L_{3}+L_{4}$. 


\section{References}

[1] L. Ambrosio, N. Fusco, and D. Pallara. Functions of bounded variation and free discontinuity problems. Oxford Mathematical Monographs. The Clarendon Press Oxford University Press, New York, 2000.

[2] S. Bianchini and R. M. Colombo. On the stability of the standard Riemann semigroup. Proc. Amer. Math. Soc., 130(7):1961-1973 (electronic), 2002.

[3] F. Bouchut and B. Perthame. Kružkov's estimates for scalar conservation laws revisited. Trans. Amer. Math. Soc., 350(7):2847-2870, 1998.

[4] G.-Q. Chen and K. H. Karlsen. Quasilinear anisotropic degenerate parabolic equations with time-space dependent diffusion coefficients. Commun. Pure Appl. Anal., 4(2):241-266, 2005.

[5] R. M. Colombo and G. Guerra. Hyperbolic balance laws with a non local source. Communications in Partial Differential Equations, 32(12):1917-1939, 2007.

[6] C. M. Dafermos. Polygonal approximations of solutions of the initial value problem for a conservation law. J. Math. Anal. Appl., 38:33-41, 1972.

[7] C. M. Dafermos. Hyperbolic conservation laws in continuum physics, volume 325 of Grundlehren der Mathematischen Wissenschaften [Fundamental Principles of Mathematical Sciences]. Springer-Verlag, Berlin, second edition, 2005.

[8] M. Di Francesco. Initial value problem and relaxation limits of the Hamer model for radiating gases in several space variables. NoDEA, 13(5-6):531-562, 2007.

[9] K. H. Karlsen and N. H. Risebro. On the uniqueness and stability of entropy solutions of nonlinear degenerate parabolic equations with rough coefficients. Discrete Contin. Dyn. Syst., 9(5):1081-1104, 2003.

[10] S. Kawashima and S. Nishibata. Cauchy problem for a model system of the radiating gas: weak solutions with a jump and classical solutions. Math. Models Methods Appl. Sci., 9(1):69-91, 1999.

[11] S. Kawashima and S. Nishibata. Shock waves for a model system of the radiating gas. SIAM J. Math. Anal., 30(1):95-117 (electronic), 1999.

[12] S. N. Kružkov. First order quasilinear equations with several independent variables. Mat. Sb. (N.S.), 81 (123):228-255, 1970.

[13] C. Lattanzio and P. Marcati. Global well-posedness and relaxation limits of a model for radiating gas. J. Differential Equations, 190(2):439-465, 2003.

[14] C. Lin, J.-F. Coulombel, and T. Goudon. Shock profiles for non-equilibrium radiating gases. Phys. D, 218(1):83-94, 2006.

[15] H. Liu and E. Tadmor. Critical thresholds in a convolution model for nonlinear conservation laws. SIAM J. Math. Anal., 33(4):930-945 (electronic), 2001.

[16] B. J. Lucier. A moving mesh numerical method for hyperbolic conservation laws. Math. Comp., 46(173):59-69, 1986.

[17] D. Serre. $L^{1}$-stability of constants in a model for radiating gases. Commun. Math. Sci., 1(1):197-205, 2003.

[18] A. I. Vol'pert and S. I. Hudjaev. Analysis in classes of discontinuous functions and equations of mathematical physics, volume 8 of Mechanics: Analysis. Martinus Nijhoff Publishers, Dordrecht, 1985. 